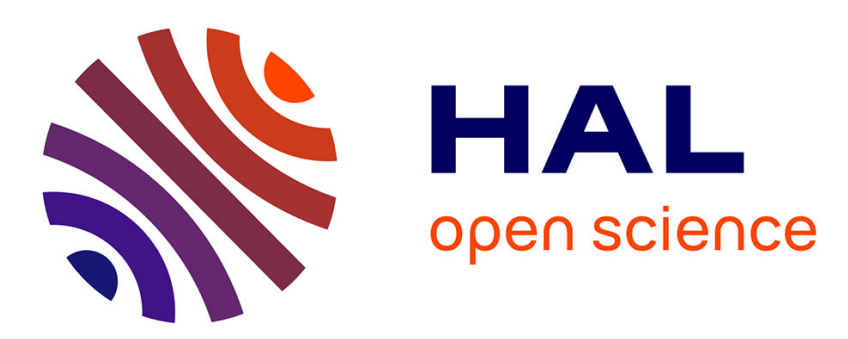

\title{
Pulsating travelling fronts: Asymptotics and homogenization regimes
}

\author{
Mohammad El Smaily
}

\section{To cite this version:}

Mohammad El Smaily. Pulsating travelling fronts: Asymptotics and homogenization regimes. European Journal of Applied Mathematics, 2008, 19 (04), pp.393-434. hal-00172332v5

\section{HAL Id: hal-00172332 \\ https://hal.science/hal-00172332v5}

Submitted on 21 Nov 2010

HAL is a multi-disciplinary open access archive for the deposit and dissemination of scientific research documents, whether they are published or not. The documents may come from teaching and research institutions in France or abroad, or from public or private research centers.
L'archive ouverte pluridisciplinaire HAL, est destinée au dépôt et à la diffusion de documents scientifiques de niveau recherche, publiés ou non, émanant des établissements d'enseignement et de recherche français ou étrangers, des laboratoires publics ou privés. 


\title{
Pulsating travelling fronts: Asymptotics and homogenization regimes
}

\author{
Mohammad El Smaily* \\ Université Aix-Marseille III, LATP, Faculté des Sciences et Techniques, \\ Avenue Escadrille Normandie-Niemen, F-13397 Marseille Cedex 20, France.
}

28 March 2008

\begin{abstract}
$\mathcal{A}$ bstract. This paper is concerned with some nonlinear propagation phenomena for reactionadvection-diffusion equations with Kolmogrov-Petrovsky-Piskunov (KPP) type nonlinearities in general periodic domains or in infinite cylinders with oscillating boundaries. Having a variational formula for the minimal speed of propagation involving eigenvalue problems ( proved in Berestycki, Hamel and Nadirashvili [3]), we consider the minimal speed of propagation as a function of diffusion factors, reaction factors and periodicity parameters. There we study the limits, the asymptotic behaviors and the variations of the considered functions with respect to these parameters. Section 8 deals with homogenization problem as an application of the results in the previous sections in order to find the limit of the minimal speed when the periodicity cell is very small.
\end{abstract}

\section{Introduction}

This paper is a continuation in the study of the propagation phenomena of pulsating travelling fronts in a periodic framework corresponding to reaction-advection-diffusion equations with heterogenous KPP (Kolmogrov, Petrovsky and Piskunov) nonlinearities. We will precisely describe the heterogenous-periodic setting, recall the extended notion of pulsating travelling fronts, and then we move to announce the main results. Let us first recall some of the basic features of the homogenous KPP equations.

Consider the Fisher-KPP equation:

$$
u_{t}-\Delta u=f(u) \quad \text { in } \mathbb{R}^{N} .
$$

It was introduced in the celebrated papers of Fisher (1937) and in 19 originally motivated by models in biology. Here, the main assumption is that $f$ is, say, a $C^{1}$ function satisfying

$$
\begin{gathered}
f(0)=f(1)=0, f^{\prime}(1)<0, f^{\prime}(0)>0 \\
f>0 \text { in }(0,1), f<0 \text { in }(1,+\infty), \\
f(s) \leq f^{\prime}(0) s, \forall s \in[0,1]
\end{gathered}
$$

As examples of such nonlinearities, we have: $f(s)=s(1-s)$ and $f(s)=s\left(1-s^{2}\right)$.

\footnotetext{
${ }^{*}$ E-mail: mohammad.el-smaily@etu.univ-cezanne.fr
} 
The important feature in (1.1) is that this equation has a family of planar travelling fronts. These are solutions of the form

$$
\left\{\begin{array}{l}
\forall(t, x) \in \mathbb{R} \times \mathbb{R}^{N}, u(t, x)=\phi(x \cdot e+c t) \\
\phi(-\infty)=0 \text { and } \phi(+\infty)=1
\end{array}\right.
$$

where $e \in \mathbb{R}^{N}$ is a fixed vector of unit norm which is the direction of propagation, and $c>0$ is the speed of the front. The function $\phi: \mathbb{R} \mapsto \mathbb{R}$ satisfies

$$
\left\{\begin{array}{l}
-\phi^{\prime \prime}+c \phi=f(\phi) \\
\phi(-\infty)=0 \text { and } \phi(+\infty)=1
\end{array}\right.
$$

In the original paper of Kolmogorov, Petrovsky and Piskunov, it was proved that, under the above assumptions, there is a threshold value $c^{*}=2 \sqrt{f^{\prime}(0)}>0$ for the speed $c$. Namely, no fronts exist for $c<c^{*}$, and, for each $c \geq c^{*}$, there is a unique front of the type (1.4 1.5). Uniqueness is up to shift in space or time variables.

Later, the homogenous setting was extended to a general heterogenous periodic one. The heterogenous character appeared both in the reaction-advection-diffusion equation and in the underlying domain. The general form of these equations is

$$
\left\{\begin{array}{l}
u_{t}=\nabla \cdot(A(z) \nabla u)+q(z) \cdot \nabla u+f(z, u), t \in \mathbb{R}, z \in \Omega \\
\nu \cdot A \nabla u(t, z)=0, t \in \mathbb{R}, z \in \partial \Omega
\end{array}\right.
$$

where $\nu(z)$ is the unit outward normal on $\partial \Omega$ at the point $z$.

The propagation phenomena attached with equation (1.6) has been widely studied in many papers. Several properties of pulsating fronts in periodic media and their speed of propagation were given in several papers ( Berestycki, Hamel [2], Berestycki, Hamel, Nadirashvili [3], and Berestycki, Hamel, Roques [5, 6] and Xin [36]). In section 2, we will recall the periodic framework and some known results which motivate our study. The main results of this paper are presented in sections 3 to 6 .

\section{The periodic framework}

\subsection{Pulsating travelling fronts in periodic domains}

In this section, we introduce the general setting with the precise assumptions. Concerning the domain, let $N \geq 1$ be the space dimension, and let $d$ be an integer so that $1 \leq d \leq N$. For an element $z=\left(x_{1}, x_{2}, \cdots, x_{d}, x_{d+1}, \cdots, x_{N}\right) \in \mathbb{R}^{N}$, we call $x=\left(x_{1}, x_{2}, \cdots, x_{d}\right)$ and $y=$ $\left(x_{d+1}, \cdots, x_{N}\right)$ so that $z=(x, y)$. Let $L_{1}, \cdots, L_{d}$ be $d$ positive real numbers, and let $\Omega$ be a $C^{3}$ nonempty connected open subset of $\mathbb{R}^{N}$ satisfying

$$
\left\{\begin{array}{l}
\exists R \geq 0 ; \forall(x, y) \in \Omega,|y| \leq R, \\
\forall\left(k_{1}, \cdots, k_{d}\right) \in L_{1} \mathbb{Z} \times \cdots \times L_{d} \mathbb{Z}, \quad \Omega=\Omega+\sum_{k=1}^{d} k_{i} e_{i},
\end{array}\right.
$$

where $\left(e_{i}\right)_{1 \leq i \leq N}$ is the canonical basis of $\mathbb{R}^{N}$. In particular, since $d \geq 1$, the set $\Omega$ is unbounded. In this periodic situation, we give the following definitions: 
Definition 2.1 (Periodicity cell) The set $C=\left\{(x, y) \in \Omega ; x_{1} \in\left(0, L_{1}\right), \cdots, x_{d} \in\left(0, L_{d}\right)\right\}$ is called the periodicity cell of $\Omega$.

Definition 2.2 (L-periodic flows ) A field $w: \Omega \rightarrow \mathbb{R}^{N}$ is said to be L-periodic with respect to $x$ if $w\left(x_{1}+k_{1}, \cdots, x_{d}+k_{d}, y\right)=w\left(x_{1}, \cdots, x_{d}, y\right)$ almost everywhere in $\Omega$, and for all $k=\left(k_{1}, \cdots, k_{d}\right) \in \prod_{i=1}^{d} L_{i} \mathbb{Z}$.

Before going further on, we point out that this framework includes several types of simpler geometrical configurations. The case of the whole space $\mathbb{R}^{N}$ corresponds to $d=N$, where $L_{1}, \ldots, L_{N}$ are any positive numbers. The case of the whole space $\mathbb{R}^{N}$ with a periodic array of holes can also be considered. The case $\mathrm{d}=1$ corresponds to domains which have only one unbounded dimension, namely infinite cylinders which may be straight or have oscillating periodic boundaries, and which may or may not have periodic holes. The case $2 \leq d \leq N-1$ corresponds to infinite slabs.

We are concerned with propagation phenomena for the reaction-advection-diffusion equation (1.6) set in the periodic domain $\Omega$. Such equations arise in combustion models for flame propagation (see [27], [31] and [37]), as well as in models in biology and for population dynamics of a species (see [14], 18], 20] and [28]). These equations are used in modeling the propagation of a flame or of an epidemics in a periodic heterogenous medium. The passive quantity $u$ typically stands for the temperature or a concentration which diffuses in a periodic excitable medium. However, in some sections we will ignore the advection and deal only with reaction-diffusion equations.

Let us now detail the assumptions concerning the coefficients in (1.6). First, the diffusion matrix $A(x, y)=\left(A_{i j}(x, y)\right)_{1 \leq i, j \leq N}$ is a symmetric $C^{2, \delta}(\bar{\Omega})$ (with $\delta>0$ ) matrix field satisfying

$$
\left\{\begin{array}{l}
A \text { is } L \text {-periodic with respect to } x \\
\exists 0<\alpha_{1} \leq \alpha_{2}, \forall(x, y) \in \Omega, \forall \xi \in \mathbb{R}^{N} \\
\alpha_{1}|\xi|^{2} \leq \sum_{1 \leq i, j \leq N} A_{i j}(x, y) \xi_{i} \xi_{j} \leq \alpha_{2}|\xi|^{2}
\end{array}\right.
$$

The boundary condition $\nu \cdot A \nabla u(x, y)=0$ stands for $\sum_{1 \leq i, j \leq N} \nu_{i}(x, y) A_{i j}(x, y) \partial_{x_{j}} u(t, x, y)$, and $\nu$ stands for the unit outward normal on $\partial \Omega$. We note that when $A$ is the identity matrix, then this boundary condition reduces to the usual Neumann condition $\partial_{\nu} u=0$.

The underlying advection $q(x, y)=\left(q_{1}(x, y), \cdots, q_{N}(x, y)\right)$ is a $C^{1, \delta}(\bar{\Omega})$ (with $\left.\delta>0\right)$ vector field satisfying

$$
\left\{\begin{array}{l}
q \quad \text { is } L-\text { periodic with respect to } x \\
\nabla \cdot q=0 \text { in } \bar{\Omega} \\
q \cdot \nu=0 \text { on } \partial \Omega \\
\forall 1 \leq i \leq d, \quad \int_{C} q_{i} d x d y=0 .
\end{array}\right.
$$


Concerning the nonlinearity, let $f=f(x, y, u)$ be a nonnegative function defined in $\bar{\Omega} \times[0,1]$, such that

$$
\left\{\begin{array}{l}
f \geq 0, f \text { is } L \text {-periodic with respect to } x, \text { and of class } C^{1, \delta}(\bar{\Omega} \times[0,1]), \\
\forall(x, y) \in \bar{\Omega}, \quad f(x, y, 0)=f(x, y, 1)=0, \\
\exists \rho \in(0,1), \forall(x, y) \in \bar{\Omega}, \forall 1-\rho \leq s \leq s^{\prime} \leq 1, f(x, y, s) \geq f\left(x, y, s^{\prime}\right), \\
\forall s \in(0,1), \exists(x, y) \in \bar{\Omega} \text { such that } f(x, y, s)>0, \\
\forall(x, y) \in \bar{\Omega}, \quad f_{u}^{\prime}(x, y, 0)=\lim _{u \rightarrow 0^{+}} \frac{f(x, y, u)}{u}>0,
\end{array}\right.
$$

with the additional assumption

$$
\forall(x, y, s) \in \bar{\Omega} \times(0,1), \quad 0<f(x, y, s) \leq f_{u}^{\prime}(x, y, 0) \times s .
$$

We denote by $\zeta(x, y):=f_{u}^{\prime}(x, y, 0)$, for each $(x, y) \in \bar{\Omega}$.

The set of such nonlinearities contains two particular types of functions:

- The homogeneous (KPP) type: $f(x, y, u)=g(u)$, where $g$ is a $C^{1, \delta}$ function that satisfies:

$$
g(0)=g(1)=0, g>0 \text { on }(0,1), g^{\prime}(0)>0, g^{\prime}(1)<0 \text { and } 0<g(s) \leq g^{\prime}(0) s \text { in }(0,1) .
$$

- Another type of such nonlinearities consists of functions $f(x, y, u)=h(x, y) \cdot \tilde{f}(u)$, such that $\tilde{f}$ is of the previous type, while $h$ lies in $C^{1, \delta}(\bar{\Omega}), L$-periodic with respect to $x$, and positive in $\bar{\Omega}$.

Having this periodic framework, the notions of travelling fronts and propagation were extended, in [2], [3], [18], 26] [28], 229], and [34] as follows:

Definition 2.3 Let $e=\left(e^{1}, \cdots, e^{d}\right)$ be an arbitrarily given vector in $\mathbb{R}^{d}$. A function $u=u(t, x, y)$ is called a pulsating travelling front propagating in the direction of $e$ with an effective speed $c \neq 0$, if $u$ is a classical solution of

$$
\left\{\begin{array}{l}
u_{t}=\nabla \cdot(A(x, y) \nabla u)+q(x, y) \cdot \nabla u+f(x, y, u), t \in \mathbb{R},(x, y) \in \Omega, \\
\nu \cdot A \nabla u(t, x, y)=0, t \in \mathbb{R},(x, y) \in \partial \Omega, \\
\forall k \in \prod_{i=1}^{d} L_{i} \mathbb{Z}, \forall(t, x, y) \in \mathbb{R} \times \bar{\Omega}, \quad u\left(t-\frac{k \cdot e}{c}, x, y\right)=u(t, x+k, y), \\
\quad \lim _{x \cdot e \rightarrow-\infty} u(t, x, y)=0, \text { and } \lim _{x \cdot e \rightarrow+\infty} u(t, x, y)=1, \\
0 \leq u \leq 1,
\end{array}\right.
$$

where the above limits hold locally in $t$ and uniformly in $y$ and in the directions of $\mathbb{R}^{d}$ which are orthogonal to $e$.

\subsection{Some important known results concerning the propagation phenomena in a periodic framework}

Under the assumptions (2.1), (2.2), (2.3), (2.4) and (2.5) set in the previous subsection, Berestycki and Hamel [2] proved that: having a pre-fixed unit vector $e \in \mathbb{R}^{d}$, there exists $c^{*}(e)>0$ such 
that pulsating travelling fronts propagating in the direction $e$ (i.e satisfying (2.6)) with a speed of propagation $c$ exist if and only if $c \geq c^{*}(e)$; moreover, the pulsating fronts (within a speed $c \geq c^{*}(e)$ ) are increasing in the time $t$. The value $c^{*}(e)=c_{\Omega, A, q, f}^{*}(e)$ is called the minimal speed of propagation in the direction of $e$. Other nonlinearities have been considered in the cases of the whole space $\mathbb{R}^{N}$ or in the general periodic framework (see [2], [28], [29], [32], [33], [34], [35]).

Having the threshold value $c_{\Omega, A, q, f}^{*}(e)$, our paper aims to study the limits, the asymptotic behaviors, and the variations of some parametric quantities. These parametric quantities involve the parametric speeds of propagation of different reaction-advection-diffusion problems within a diffusion factor $\varepsilon>0$, a reaction factor $B>0$, or a periodicity parameter $L$. Thus, it is important to have a variational characterization which shows the dependance of the minimal speed of propagation on the coefficients $A, q$ and $f$ and on the geometry of the domain $\Omega$. In this context, Berestycki, Hamel, and Nadirashvili [3] gave such a formulation for $c_{\Omega, A, q, f}^{*}(e)$ involving elliptic eigenvalue problems. We recall this variational characterization in the following theorem:

Theorem 2.4 (Berestycki, Hamel, and Nadirashvili [3]) Let e be a fixed unit vector in $\mathbb{R}^{d}$. Let $\tilde{e}=(e, 0, \ldots, 0) \in \mathbb{R}^{N}$. Assume that $\Omega, A$ and $f$ satisfy (2.1), (2.2), (2.4), and (2.5). The minimal speed $c^{*}(e)=c_{\Omega, A, q, f}^{*}(e)$ of pulsating fronts solving (2.6) and propagating in the direction of $e$ is given by

$$
c^{*}(e)=c_{\Omega, A, q, f}^{*}(e)=\min _{\lambda>0} \frac{k(\lambda)}{\lambda},
$$

where $k(\lambda)=k_{\Omega, e, A, q, \zeta}(\lambda)$ is the principal eigenvalue of the operator $L_{\Omega, e, A, q, \zeta, \lambda}$ which is defined by

$$
\begin{aligned}
L_{\Omega, e, A, q, \zeta, \lambda} \psi:= & \nabla \cdot(A \nabla \psi)-2 \lambda \tilde{e} \cdot A \nabla \psi+q \cdot \nabla \psi \\
& +\left[\lambda^{2} \tilde{e} A \tilde{e}-\lambda \nabla \cdot(A \tilde{e})-\lambda q \cdot \tilde{e}+\zeta\right] \psi
\end{aligned}
$$

acting on the set

$$
E=\left\{\psi \in C^{2}(\bar{\Omega}), \psi \text { is L-periodic with respect to } x \text { and } \nu \cdot A \nabla \psi=\lambda(\nu A \tilde{e} \psi) \text { on } \partial \Omega\right\} .
$$

The proof of formula (2.7) is based on methods developed in [2], [7] and [9]. These are techniques of sub and super-solutions, regularizing and approximations in bounded domains.

We note that in formula (2.7), the value of the minimal speed $c^{*}(e)$ is given in terms of the direction $e$, the domain $\Omega$, and the coefficients $A, q$ and $f_{u}^{\prime}(., ., 0)$. Moreover, it is important to notice that the dependence of $c^{*}(e)$ on the nonlinearity $f$ is only through the derivative of $f$ with respect to $u$ at $u=0$.

Before going further on, let us mention that formula (2.7) extends some earlier results about front propagation. When $\Omega=\mathbb{R}^{N}, A=I d$ and $f=f(u)$ (with $f(u) \leq f^{\prime}(0) u$ in $[0,1]$ ), formula (2.7) then reduces to the well-known KPP formula $c^{*}(e)=2 \sqrt{f^{\prime}(0)}$. That is the value of the minimal speed of propagation of planar fronts for the homogenous reaction-diffusion equation: $u_{t}-\Delta u=f(u)$ in $\mathbb{R}^{N}$.

The above variational characterization of the minimal speed of propagation of pulsating fronts in general periodic excitable media will play the main role in studying the dependence of the minimal speed $c^{*}(e)=c_{\Omega, A, q, f}^{*}(e)$ on the coefficients of reaction, diffusion, advection and on the geometry of the domain. In this context, we have:

\footnotetext{
${ }^{1}$ In fact, the uniqueness, up to multiplication by a non-zero real number, of the first eigenvalue function of $L_{\mathbb{R}^{N}, e, I d, f^{\prime}(0), \lambda} \psi=k(\lambda) \psi$ together with this particular situation, yield that the principal eigenfunction $\psi$ is constant and $k(\lambda)=\lambda^{2}+f^{\prime}(0)$ for all $\lambda>0$. Therefore by (2.7), we have $c^{*}(e)=\min _{\lambda>0}\left(\lambda+\frac{f^{\prime}(0)}{\lambda}\right)=2 \sqrt{f^{\prime}(0)}$.
} 
Theorem 2.5 (Berestycki, Hamel, Nadirashvili [3]) Under the assumptions (2.1), (2.2), and (2.3) on $\Omega, A$, and $q$, let $f=f(x, y, u)$ [respectively $g=g(x, y, u)$ ] be a nonnegative nonlinearity satisfying (2.4) and (2.5). Let e be a fixed unit vector in $\mathbb{R}^{d}$, where $1 \leq d \leq N$,

a) If $f_{u}^{\prime}(x, y, 0) \leq g_{u}(x, y, 0)$ for all $(x, y) \in \bar{\Omega}$, then

$$
c_{\Omega, A, q, f}^{*}(e) \leq c_{\Omega, A, q, g}^{*}(e) .
$$

Moreover if $f_{u}^{\prime}(x, y, 0) \leq, \not \equiv g_{u}^{\prime}(x, y, 0)$ in $\bar{\Omega}$, then $c_{\Omega, A, q, f}^{*}(e)<c_{\Omega, A, q, g}^{*}(e)$.

b) The map $B \mapsto c_{\Omega, A, q, B f}^{*}(e)$ is increasing in $B>0$ and

$$
\limsup _{B \rightarrow+\infty} \frac{c_{\Omega, A, q, B f}^{*}(e)}{\sqrt{B}}<+\infty
$$

Furthermore, if $\Omega=\mathbb{R}^{N}$ or if $\nu A \tilde{e} \equiv 0$ on $\partial \Omega$, then $\liminf _{B \rightarrow+\infty} \frac{c_{\Omega, A, q, B f}^{*}(e)}{\sqrt{B}}>0$.

c)

$$
c_{\Omega, A, q, f}^{*}(e) \leq\left\|(q . \tilde{e})^{-}\right\|_{\infty}+2 \sqrt{\max _{(x, y) \in \bar{\Omega}} \zeta(x, y)} \sqrt{\max _{(x, y) \in \bar{\Omega}} \tilde{e} A(x, y) \tilde{e}}
$$

where $\left\|(q . \tilde{e})^{-}\right\|_{\infty}=\max _{(x, y) \in \bar{\Omega}}(q(x, y) . \tilde{e})^{-}$and $s^{-}=\max (-s, 0)$ for each $s \in \mathbb{R}$. Furthermore, the equality holds in (2.9) if and only if $\tilde{e} A \tilde{e}$ and $\zeta$ are constant, q.e $\equiv \nabla .(A \tilde{e}) \equiv 0$ in $\bar{\Omega}$ and $\nu . A \tilde{e}=0$ on $\partial \Omega($ in the case when $\partial \Omega \neq \emptyset)$.

d) Assume furthermore that $f=f(u)$ and $q \equiv 0$ in $\bar{\Omega}$, then the $\operatorname{map} \beta \mapsto c_{\Omega, \beta A, 0, f}^{*}(e)$ is increasing in $\beta>0$.

As a corollary of (2.9), we see that $\limsup _{M \rightarrow+\infty} \frac{c_{\Omega, M A, q, f}^{*}(e)}{\sqrt{M}} \leq C$ where $C$ is a positive constant. Furthermore, part d) implies that a larger diffusion speeds up the propagation in the absence of the advection field.

We mention that the existence of pulsating travelling fronts in space-time periodic media was proved in Nolen, Xin [23, 24], Nolen, Rudd, Xin [25] and recently in Nadin [21, 22]. In [22], Nadin characterized the minimal speed of propagation and he studied the influence of the diffusion, the amplitude of the reaction term and the drift on the characterized speed.

After reviewing some results in the study of the KPP propagation phenomena in a periodic framework, we pass now to announce new results concerning the limiting behavior of the minimal speed of propagation within a small (resp. large) diffusion and reaction coefficients (in some particular situations of the general periodic framework) and we will study the minimal speed as a function of the period of the coefficients in the KPP reaction-diffusion-advection (or reactiondiffusion) equation in the case where $\Omega=\mathbb{R}^{N}$. The proofs will be shown in details in section 7 . The announced results will be applied to find the homogenization limit of the minimal speeds of propagation. We believe that this limit might help to find the homogenized equation in the "KPP" periodic framework (see section 8 for more details). 


\section{The minimal speed within small diffusion factors or within large period coefficients}

In this section, our problem is a reaction-diffusion equation with absence of advection terms:

$$
\left\{\begin{array}{l}
u_{t}=\beta \nabla \cdot(A(x, y) \nabla u)+f(x, y, u), t \in \mathbb{R},(x, y) \in \Omega \\
\nu \cdot A \nabla u(t, x, y)=0, t \in \mathbb{R},(x, y) \in \partial \Omega
\end{array}\right.
$$

where $\beta>0$.

We mention that (3.1) is a reaction-diffusion problem within a diffusion matrix $\beta A$. Let $e$ be a unit direction in $\mathbb{R}^{d}$. Under the assumptions (2.1), (2.2), (2.4) and (2.5), for each $\beta>0$, there corresponds a minimal speed of propagation $c_{\Omega, \beta A, 0, f}^{*}(e)$ so that a pulsating front with a speed $c$ and satisfying (3.1) exists if and only if $c \geq c_{\Omega, \beta A, 0, f}^{*}(e)$.

Referring to part c) of Theorem 2.5, one gets $0<c_{\Omega, \beta A, 0, f}^{*}(e) \leq 2 \sqrt{\beta} \sqrt{M_{0} M}$, for any $\beta>0$, where $M_{0}=\max _{(x, y) \in \bar{\Omega}} \zeta(x, y)$ and $M=\max _{(x, y) \in \bar{\Omega}} \tilde{e} A(x, y) \tilde{e}$.

Consequently, there exists $C>0$ and independent of $\beta$ such that

$$
\forall \beta>0,0<\frac{c_{\Omega, \beta A, 0, f}^{*}(e)}{\sqrt{\beta}} \leq C .
$$

The inequality (3.2) leads us to investigate the limits of $\frac{c_{\Omega, \beta A, 0, f}^{*}(e)}{\sqrt{\beta}}$ as $\beta \rightarrow 0$ and as $\beta \rightarrow+\infty$. The following theorem gives the precise limit when the diffusion factor tends to zero. However, it will not be announced in the most general periodic setting. We will describe the situation before the statement of the theorem:

The domain will be in the form $\Omega=\mathbb{R} \times \omega \subseteq \mathbb{R}^{N}$, where $\omega \subseteq \mathbb{R}^{d} \times \mathbb{R}^{N-d-1}(d \geq 0)$. If $d=0$, then $\omega$ is a $C^{3}$ connected, open bounded subset of $\mathbb{R}^{N-1}$. While, in the case where $1 \leq d \leq N-1, \quad \omega$ is a $\left(L_{1}, \ldots, L_{d}\right)$-periodic open domain of $\mathbb{R}^{N-1}$ which satisfies (2.1); and hence, $\Omega$ is a $\left(l, L_{1}, \ldots, L_{d}\right)$-periodic subset of $\mathbb{R}^{N}$ that satisfies (2.1) with $l>0$ and arbitrary. An element of $\Omega=\mathbb{R} \times \omega$ will be represented as $z=(x, y)$ where $x \in \mathbb{R}$ and $y \in \omega \subseteq \mathbb{R}^{d} \times \mathbb{R}^{N-1-d}$.

The nonlinearity $f=f(x, y, u)$, in this section, is a KPP nonlinearity defined on $\bar{\Omega} \times[0,1]$ that satisfies

$$
\left\{\begin{array}{l}
f \geq 0, \text { and of class } C^{1, \delta}(\mathbb{R} \times \bar{\omega} \times[0,1]), \\
f \text { is }\left(l, L_{1}, \ldots, L_{d}\right) \text {-periodic with respect to }\left(x, y_{1}, \ldots, y_{d}\right), \text { when } d \geq 1, \\
f \text { is } l \text {-periodic in } x, \text { when } d=0, \\
\forall(x, y) \in \bar{\Omega}=\mathbb{R} \times \bar{\omega}, f(x, y, 0)=f(x, y, 1)=0 \\
\exists \rho \in(0,1), \forall(x, y) \in \bar{\Omega}, \forall 1-\rho \leq s \leq s^{\prime} \leq 1, f(x, y, s) \geq f\left(x, y, s^{\prime}\right), \\
\forall s \in(0,1), \quad \exists(x, y) \in \bar{\Omega} \text { such that } f(x, y, s)>0
\end{array}\right.
$$

together with the assumptions

$$
\left\{\begin{array}{l}
f_{u}^{\prime}(x, y, 0) \text { depends only on } y \text {; we denote by } \zeta(y)=f_{u}^{\prime}(x, y, 0), \forall(x, y) \in \bar{\Omega} \\
\forall(x, y) \in \bar{\Omega}=\mathbb{R} \times \bar{\omega}, \quad f_{u}^{\prime}(x, y, 0)=\zeta(y)>0 \\
\forall(x, y, s) \in \bar{\Omega} \times(0,1), \quad 0<f(x, y, s) \leq \zeta(y) s
\end{array}\right.
$$


Notice that $f_{u}^{\prime}(x, y, u)$ is assumed to depend only on $y$, but $f(x, y, u)$ may depend on $x$.

Lastly, concerning the diffusion matrix, $A(x, y)=A(y)=\left(A_{i j}(y)\right)_{1 \leq i, j \leq N}$ is a $C^{2, \delta}(\bar{\Omega})$ (with $\delta>0)$ symmetric matrix field whose entries are depending only on $y$, and satisfying

$$
\left\{\begin{array}{l}
A \text { is }\left(L_{1}, \ldots, L_{d}\right) \text {-periodic with respect to }\left(y_{1}, \ldots, y_{d}\right), \\
\exists 0<\alpha_{1} \leq \alpha_{2}, \forall y \in \omega, \forall \xi \in \mathbb{R}^{N} \\
\alpha_{1}|\xi|^{2} \leq \sum A_{i j}(y) \xi_{i} \xi_{j} \leq \alpha_{2}|\xi|^{2}
\end{array}\right.
$$

Theorem 3.1 Let $e=(1,0, \ldots, 0) \in \mathbb{R}^{N}$ and $\varepsilon>0$. Let $\Omega=\mathbb{R} \times \omega \subseteq \mathbb{R}^{N}$ satisfy the form described in the previous page. Under the assumptions (3.3), (3.4), and (3.5), consider the reaction-diffusion equation

$$
\left\{\begin{aligned}
u_{t}(t, x, y) & =\varepsilon \nabla \cdot(A(y) \nabla u)(t, x, y)+f(x, y, u), \text { for }(t, x, y) \in \mathbb{R} \times \Omega, \\
\nu \cdot A \nabla u & =0 \text { on } \mathbb{R} \times \mathbb{R} \times \partial \omega .
\end{aligned}\right.
$$

Assume, furthermore, that $A$ and $f$ satisfy one of the following two alternatives:

$$
\left\{\begin{array}{l}
\exists \alpha>0, \forall y \in \omega, A(y) e=\alpha e, \\
f_{u}^{\prime}(x, y, 0)=\zeta(y), \text { for all }(x, y) \in \bar{\Omega},
\end{array}\right.
$$

or

$$
\left\{\begin{array}{l}
f_{u}^{\prime}(x, y, 0)=\zeta \text { is constant, } \\
\forall y \in \omega, A(y) e=\alpha(y) e, \text { where } \\
y \mapsto \alpha(y) \text { is a positive, }\left(L_{1}, \ldots, L_{d}\right) \text {-periodic function over } \bar{\omega} .
\end{array}\right.
$$

Then,

$$
\lim _{\varepsilon \rightarrow 0^{+}} \frac{c_{\Omega, \varepsilon A, 0, f}^{*}(e)}{\sqrt{\varepsilon}}=2 \sqrt{\max _{\bar{\omega}} \zeta} \sqrt{\max _{\bar{\omega}} e A e} .
$$

Before going further on, we mention that the family of domains for which Theorem 3.1 holds is wide. An infinite cylinder $\mathbb{R} \times B_{\mathbb{R}^{N-1}}\left(y_{0}, R\right)$ (where $R>0$, and $B_{\mathbb{R}^{N-1}}\left(y_{0}, R\right)$ is the Euclidian ball of center $y_{0}$ and radius $R$ ) is an archetype of such domains. In these cylinders, $\omega=B_{\mathbb{R}^{N-1}}\left(y_{0}, R\right), l$ is any positive real number, and $d=0$. The whole space $\mathbb{R}^{N}$ is another archetype of the domain $\Omega$ where $d=N-1, \omega=\mathbb{R}^{N-1}$, and $\left\{l, L_{1}, \ldots, L_{d}\right\}$ is any family of positive real numbers.

Remark 3.2 In Theorem 3.1, the domain $\Omega=\mathbb{R} \times \omega$ is invariant in the direction of $e=(1,0 \ldots, 0)$ which is parallel to $A e$ (in both cases (3.7) and (3.8)). Also, the assumption that the entries of $A$ do not depend on $x$, yields that $\nabla \cdot(A e) \equiv 0$ over $\Omega$. On the other hand, it is easy to find a diffusion matrix $A$ and a nonlinearity $f$ which satisfy, together, the assumptions of Theorem 3.1 while one of $e A e(y)$ and $\zeta(y)$ is not constant. Referring to part c) of Theorem 2.5, one obtains:

$$
\forall \varepsilon>0, \quad 0<\frac{c_{\Omega, \varepsilon A, 0, f}^{*}(e)}{\sqrt{\varepsilon}} \supsetneqq 2 \sqrt{\max _{y \in \bar{\omega}} \zeta(y)} \sqrt{\max _{y \in \bar{\omega}} e A e(y)} .
$$

However, Theorem 3.1 implies that

$$
\lim _{\varepsilon \rightarrow 0^{+}} \frac{c_{\Omega, \varepsilon A, 0, f}^{*}(e)}{\sqrt{\varepsilon}}=2 \sqrt{\max _{y \in \bar{\omega}} \zeta(y)} \sqrt{\max _{y \in \bar{\omega}} e A e(y)} .
$$

On the other hand, if $\Omega=\mathbb{R} \times \omega$ as in Theorem 3.1, $A=I d$ and $f=f(u)$, Theorem 2.5 yields that $c_{\Omega, \varepsilon I d, 0, f}^{*}(e)=2 \sqrt{\varepsilon} \sqrt{f^{\prime}(0)}$, for all $\varepsilon>0$. 
In the same context, one can also find the limit when the diffusion factor goes to zero, but in the presence of an advection field in the form of shear flows:

Theorem 3.3 Assume that $e=(1,0, \cdots, 0) \in \mathbb{R}^{N}$, the domain $\Omega=\mathbb{R} \times \omega$ has the same form as in Theorem 3.1, and the coefficients $f$ and $A$ satisfy (3.9.3.4) and (3.5) respectively. Assume, furthermore, that for all $y \in \bar{\omega}$, there exists $\alpha(y)$ positive so that $A(y) e=\alpha(y)$ e in $\bar{\omega}$. Consider, in addition, an advective shear flow $q=\left(q_{1}(y), 0, \ldots, 0\right)(y \in \bar{\omega})$ which is $\left(L_{1}, \cdots, L_{d}\right)$-periodic with respect to $y$. Assume that $\varepsilon$ is a positive parameter and consider the parametric reaction-advectiondiffusion problem

$$
\left\{\begin{array}{l}
u_{t}=\varepsilon \nabla \cdot(A(y) \nabla u)+q_{1}(y) \partial_{x} u(t, x, y)+f(x, y, u), \quad t \in \mathbb{R},(x, y) \in \Omega, \\
\nu \cdot A \nabla u(t, x, y)=0, \quad t \in \mathbb{R}, \quad(x, y) \in \partial \Omega,
\end{array}\right.
$$

where $q \not \equiv 0$ over $\mathbb{R} \times \bar{\omega}$ and $q$ has a zero average. Then,

$$
\lim _{\varepsilon \rightarrow 0^{+}} c_{\Omega, \varepsilon A, q, f}^{*}(e)=\max _{y \in \bar{\omega}}\left(-q_{1}(y)\right)=\max _{\bar{\omega}}(-q \cdot e) .
$$

The situation in this result is more general than that considered in part b) of Corollary 4.5 in [4]. In details, the coefficients $A$ and $f$ can be both non-constant. Meanwhile, in the result of [4], the coefficients considered were assumed to satisfy the alternative (3.7).

After having the exact value of $\lim _{\varepsilon \rightarrow 0^{+}} \frac{c_{\Omega, \varepsilon A, 0, f}^{*}(e)}{\sqrt{\varepsilon}}$, we move now to investigate the limit of the minimal speed of propagation, considered as a function of the period of the coefficients of the reaction-diffusion equation set in the whole space $\mathbb{R}^{N}$, when the periodicity parameter tends to $+\infty$. By making some change in variables, we will find a link between this problem and Theorem 3.1:

Theorem 3.4 Let $e=(1,0, \ldots, 0) \in \mathbb{R}^{N}$. An element $z \in \mathbb{R}^{N}$ is represented as $z=(x, y) \in$ $\mathbb{R} \times \mathbb{R}^{N-1}$. Assume that $f=f(x, y, u)$ and $A=A(y)$ satisfy (3.S), (3.4) and (3.5) with $\omega=$ $\mathbb{R}^{N-1}, d=N-1$, and $l=L_{1}=\ldots=L_{N-1}=1$. (That is, the domain and the coefficients of the equation are $(1,1, \ldots, 1)$ periodic with respect to $y)$. Assume furthermore, that $A$ and $f$ satisfy either (3.7) or 3.8). For each $L>0$, and $(x, y) \in \mathbb{R}^{N}$, let $A_{L}(y)=A\left(\frac{y}{L}\right)$ and $f_{L}(x, y, u)=f\left(\frac{x}{L}, \frac{y}{L}, u\right)$. Consider the reaction-diffusion problem

$$
\begin{aligned}
u_{t}(t, x, y) & =\nabla \cdot\left(A_{L} \nabla u\right)(t, x, y)+f_{L}(x, y, u),(t, x, y) \in \mathbb{R} \times \mathbb{R}^{N} \\
& =\nabla \cdot\left(A\left(\frac{y}{L}\right) \nabla u\right)(t, x, y)+f\left(\frac{x}{L}, \frac{y}{L}, u\right),(t, x, y) \in \mathbb{R} \times \mathbb{R}^{N},
\end{aligned}
$$

whose coefficients are $(L, \ldots, L)$ periodic with respect to $(x, y) \in \mathbb{R}^{N}$. Then,

$$
\lim _{L \rightarrow+\infty} c_{\mathbb{R}^{N}}^{*}, A_{L}, 0, f_{L}(e)=2 \sqrt{\max _{y \in \mathbb{R}^{N-1}} \zeta(y)} \sqrt{\max _{y \in \mathbb{R}^{N-1}} e \cdot A e(y)} .
$$

The above theorem gives the limit of the minimal speed of propagation in the direction of $e=(1,0, \cdots, 0)$ as the periodicity parameter $L \rightarrow+\infty$. The domain is the whole space $\mathbb{R}^{N}$ which is $(L, \cdots, L)$-periodic whatever the positive number $L$. However, one can find

$$
\lim _{L \rightarrow+\infty} c_{\mathbb{R}^{N}}^{*}, A_{L}, L q_{L}, f_{L}(e)
$$

whenever $q$ is a shear flow advection. Namely, in the same manner that Theorem 3.1 implies Theorem 3.4, one can prove that Theorem 3.3 implies 
Theorem 3.5 Let $e=(1,0, \ldots, 0) \in \mathbb{R}^{N}$. Assume that $f=f(x, y, u)$ and $A=A(y)$ satisfy (3.3), (3.4) and (3.5) with $\omega=\mathbb{R}^{N-1}, d=N-1$, and $l=L_{1}=\ldots=L_{N-1}=1$. (That is, the domain and the coefficients of the equation are $(1,1, \ldots, 1)$ periodic with respect to $y$ in $\left.\mathbb{R}^{N-1}\right)$. Assume, furthermore, that for all $y \in \mathbb{R}^{N-1}$, there exists $\alpha(y)$ positive so that $A(y) e=\alpha(y)$ e in $\mathbb{R}^{N-1}$. Let $q=\left(q_{1}(y), 0, \ldots, 0\right)$ for all $y \in \mathbb{R}^{N-1}$ such that $q_{1} \not \equiv 0$ over $\mathbb{R}^{N-1}, q$ is $(1, \cdots, 1)$ - periodic with respect to $y$ and $q_{1}$ has a zero average. Then,

$$
\lim _{L \rightarrow+\infty} c_{\mathbb{R}^{N}}^{*}, A_{L}, L q_{L}, f_{L}(e)=\max _{y \in \mathbb{R}^{N-1}}\left(-q_{1}(y)\right)=\max _{\mathbb{R}^{N-1}}(-q \cdot e) .
$$

In the proof of Theorem 3.3 (which implies Theorem 3.5), the assumption that the advection $q$ is in the form of shear flows plays an important role in reducing the elliptic equation involved by the variational formula (7.13) below. Namely, since $q=\left(q_{1}(y), 0, \cdots, 0\right)$ and since $e=(1,0, \cdots, 0)$, then the terms $q(x, y) \cdot \nabla_{x, y} \psi$ and $q(x, y) \cdot e$ (in the general elliptic equation) become equal to $q_{1}(y) \partial_{x} \psi$ and $q_{1}(y)$ respectively. As a consequence, and due the uniqueness of the principal eigenfunction $\psi$ up to multiplication by a constant, we are able to choose $\psi$ independent of $x$, and hence, obtain a symmetric elliptic operator (without drift) whose principal eigenvalue was given by the variational formula (7.15) below (see section 0 for more details).

Remark 3.6 After the above explanations, we find that the techniques used to prove Theorem 3.3 which implies 3.5, will no longer work in the presence a general periodic advection field satisfying (2.3).

Concerning the influence of advection, we mention that the limit of $\frac{c_{\Omega, A, B q, f}^{*}(e)}{B}$ as $B \rightarrow+\infty$ (in the general periodic setting) is not yet given explicitly as a function of the direction $e$ and the coefficients $A, q$ and $f$. For more details one can see Theorem 4.1 in [⿶] . However, the problem of front propagation in an infinite cylinder with an underlying shear flow was widely studied in Berestycki [1], Berestycki and Nirenberg [8]. In the case of strong advection, assume that $\Omega=\mathbb{R} \times \omega$, where $\omega$ is a bounded smooth subset of $\mathbb{R}^{N-1}, q=\left(q_{1}(y), 0, \cdots, 0\right), y \in \omega$, and $f=f(u)$ is a (KPP) nonlinearity. It was proved, in Heinze [16], that

$$
\lim _{B \rightarrow+\infty} \frac{c_{\Omega, A, B q, f}^{*}(e)}{B}=\gamma
$$

where

$$
\begin{gathered}
\gamma=\sup _{\psi \in D} \int_{\omega} q_{1}(y) \psi^{2} d y \\
D=\left\{\psi \in H^{1}(w), \quad \int_{\omega}|\nabla \psi|^{2} d y \leq f^{\prime}(0), \text { and } \int_{\omega} \psi^{2} d y=1\right\} .
\end{gathered}
$$

\section{The minimal speed within large diffusion factors or within small period coefficients}

After having the limit of $c_{\Omega, \varepsilon A, 0, f}^{*}(e) / \sqrt{\varepsilon}$ as $\varepsilon \rightarrow 0^{+}$, and after knowing that this limit depends on $\max _{y \in \bar{w}} \zeta(y)$ and $\max _{y \in \bar{w}} e A e(y)$, we investigate now the limit of $c_{\Omega, M A, q, f}^{*}(e) / \sqrt{M}$ as the diffusion factor $M$ tends to $+\infty$, and we try to answer this question in a situation which is more general than that 
we considered in the previous section (in the case where the diffusion factor was going to $0^{+}$). That is in the presence of an advection field and in a domain $\Omega$ which satisfies (2.1) and which may take more forms other than those of section 3 . We will find that in the case of large diffusion, the limit will depend on $f_{C} \zeta(x, y) d x d y:=\frac{1}{|C|} \int_{C} \zeta(x, y) d x d y$ and $f_{C} \tilde{e} A \tilde{e}(x, y) d x d y:=\frac{1}{|C|} \int_{C} \tilde{e} A \tilde{e}(x, y) d x d y$, where $C$ denotes the periodicity cell of the domain $\Omega$.

Theorem 4.1 Under the assumptions (2.1) for $\Omega$, (2.5) for the advection $q$, (2.4) and (2.5) for the nonlinearity $f=f(x, y, u)$, let $e$ be any unit direction of $\mathbb{R}^{d}$. Assume that the diffusion matrix $A=A(x, y)$ satisfies (2.9) together with $\nabla \cdot A \tilde{e} \equiv 0$ over $\Omega$, and $\nu \cdot A \tilde{e}=0$ over $\partial \Omega$. For each $M>0$ and $0 \leq \gamma \leq 1 / 2$, consider the following reaction-advection-diffusion equation

$$
\left\{\begin{array}{l}
u_{t}=M \nabla \cdot(A(x, y) \nabla u)+M^{\gamma} q(x, y) \cdot \nabla u+f(x, y, u), t \in \mathbb{R},(x, y) \in \Omega, \\
\nu \cdot A \nabla u(t, x, y)=0, t \in \mathbb{R},(x, y) \in \partial \Omega .
\end{array}\right.
$$

Then

$$
\lim _{M \rightarrow+\infty} \frac{c_{\Omega, M A, M^{\gamma} q, f^{(e)}}^{*}}{\sqrt{M}}=2 \sqrt{f_{C} \tilde{e} A \tilde{e}(x, y) d x d y} \sqrt{f_{C} \zeta(x, y) d x d y},
$$

where $C$ is the periodicity cell of $\Omega$.

\section{Remarks 4.2}

- The setting in Theorem 4.1 is more general than that in Theorem 3.1, where: $\Omega=\mathbb{R} \times \omega, \tilde{e}=$ $(1,0, \ldots, 0)$, and $A \tilde{e}=\alpha(y) \tilde{e}$. Under the assumptions of Theorem 3.1, the domain $\Omega$ is invariant in the direction of $A \tilde{e}$, which is that of $\tilde{e}$. Consequently, if $\nu$ denotes the outward normal on $\partial \Omega=\mathbb{R} \times \partial \omega$, one gets $\nu \cdot A \tilde{e}=\alpha(y) \nu \cdot \tilde{e}=0$ over $\partial \Omega$, while $\nabla \cdot(A \tilde{e})=\frac{\partial}{\partial x} \alpha(y)=0$ over $\Omega$. Moreover, in Theorem 3.1, we have only reaction and diffusion terms. That is $q \equiv 0$. Therefore, considering the setting of Theorem 3.1, and taking $\beta A$ as a parametric diffusion matrix, one consequently knows the limits of $\frac{c_{\Omega, \beta A, 0, f}^{*}(e)}{\sqrt{\beta}}$ as $\beta \rightarrow 0^{+}$(Theorem 3.1) and as $\beta \rightarrow+\infty$ (Theorem 4.1).

- The other observation in Theorem 4.1 is that the limit does not depend on the advection field $q$. This may play an important role in drawing counter examples to answer many different questions. For example, the variation of the minimal speed of propagation with respect to the diffusion factor and with respect to diffusion matrices which are symmetric positive definite.

- Another important feature, in Theorem 4.1, is that the order of $M$ in the denominator of the ratio $\frac{c_{\Omega, M A, M^{\gamma} q, f}^{*}(e)}{\sqrt{M}}$ is equal to $1 / 2$. It is independent of $\gamma$. Consequently, the case where the advection is null and there is only a reaction-diffusion equation follows, in particular, from the previous theorem. That is

$$
\lim _{M \rightarrow+\infty} \frac{c_{\Omega, M A, 0, f}^{*}(e)}{\sqrt{M}}=2 \sqrt{f_{C} \tilde{e} A \tilde{e}(x, y) d x d y} \sqrt{f_{C} \zeta(x, y) d x d y} .
$$


- The previous point leads us to conclude that the presence of an advection with a factor $M^{\gamma}$, where $0 \leq \gamma \leq 1 / 2$, will have no more effect on the ratio $\frac{c_{\Omega, M A}^{*} M^{\gamma} q, f(e)}{\sqrt{M}}$ as soon as the diffusion factor $M$ gets very large.

As far as the limit of the minimal speed of propagation within small periodic coefficients in the reaction-diffusion equation is concerned, the following theorem, which mainly depends on Theorem 4.1, treats this problem:

Theorem 4.3 Let $\Omega=\mathbb{R}^{N}$. Assume that $A=A(x, y), q=q(x, y)$ and $f=f(x, y, u)$ are $(1, \ldots, 1)$-periodic with respect to $(x, y) \in \mathbb{R}^{N}$, and that they satisfy (2.9), (2.3), (2.4), and (2.5) with $L_{1}=\ldots=L_{N}=1$. Let $e$ be any unit direction of $\mathbb{R}^{N}$, such that $\nabla \cdot$ Ae $\equiv 0$ over $\mathbb{R}^{N}$. For each $L>0$, let $A_{L}(x, y)=A\left(\frac{x}{L}, \frac{y}{L}\right), q_{L}(x, y)=q\left(\frac{x}{L}, \frac{y}{L}\right)$, and $f_{L}(x, y, u)=f\left(\frac{x}{L}, \frac{y}{L}, u\right)$, where $(x, y) \in \mathbb{R}^{N}$. Consider the problem

$$
\begin{aligned}
u_{t}(t, x, y) & =\nabla \cdot\left(A_{L} \nabla u\right)(t, x, y)+q_{L} \cdot \nabla u(t, x, y)+f_{L}(x, y, u),(t, x, y) \in \mathbb{R} \times \mathbb{R}^{N}, \\
& =\nabla \cdot\left(A\left(\frac{x}{L}, \frac{y}{L}\right) \nabla u\right)(t, x, y)+q\left(\frac{x}{L}, \frac{y}{L}\right) \cdot \nabla u(t, x, y)+f\left(\frac{x}{L}, \frac{y}{L}, u\right),
\end{aligned}
$$

whose coefficients are $(L, \ldots, L)$ periodic with respect to $(x, y) \in \mathbb{R}^{N}$. Then,

$$
\lim _{L \rightarrow 0^{+}} c_{\mathbb{R}^{N}}^{*}, A_{L}, q_{L}, f_{L}(e)=2 \sqrt{f_{C} \tilde{e} A \tilde{e}(x, y) d x d y} \sqrt{f_{C} \zeta(x, y) d x d y},
$$

where, in this setting, $C=[0,1] \times \cdots \times[0,1] \subset \mathbb{R}^{N}$.

The above result gives the limit in any space dimension. It depends on the assumption $\nabla \cdot(A \tilde{e}) \equiv$ 0 in $\mathbb{R}^{N}$. However, if one takes $N=1$, and denotes the diffusion coefficient by $a=a(x), x \in \mathbb{R}$, then the previous result holds under the assumptions that $a$ satisfies $(2.2)$ and $d a / d x \equiv 0$ in $\mathbb{R}$. In other words, it holds when $a$ is a positive constant. Thus, it is be interesting to mention that, in the one-dimensional case, the above limit was given in [13] and [17] within a general diffusion coefficient (which may be not constant over $\mathbb{R}$ ). In details, assume that $f=f(x, u)=(\zeta(x)-u) u$ is a 1-periodic (KPP) nonlinearity satisfying (2.4) with (2.5), and $\mathbb{R} \ni x \mapsto a(x)$ is a 1-periodic function which satisfies $0<\alpha_{1} \leq a(x) \leq \alpha_{2}$, for all $x \in \mathbb{R}$, where $\alpha_{1}$ and $\alpha_{2}$ are two positive constants. For each $L>0$, consider the reaction-diffusion equation

$$
\partial_{t} u(t, x)=\frac{\partial}{\partial x}\left(a\left(\frac{x}{L}\right) \frac{\partial u}{\partial x}\right)(t, x)+\left[\zeta\left(\frac{x}{L}\right)-u(t, x)\right] u(t, x) \quad \text { for } \quad(t, x) \in \mathbb{R} \times \mathbb{R} .
$$

It was derived in 13 and, formally, in 17 that

$$
\lim _{L \rightarrow 0^{+}} c_{\mathbb{R}, a_{L}}^{*}, 0, f_{L}(e)=2 \sqrt{\langle a\rangle_{H} \cdot \int_{0}^{1} \zeta(x)},
$$

where $\langle a\rangle_{H}$ denotes the harmonic mean of the map $x \mapsto a(x)$ over $[0,1]$. 


\section{The minimal speed within small or large reaction coefficients}

In this section, the parameter of the reaction-advection-diffusion problem is the coefficient $B$ multiplied by the nonlinearity $f$. In fact, it follows from Theorem 1.6 in Berestycki, Hamel and Nadirashvili [3] (recalled via Theorem 2.5 in the present paper) that the map $B \mapsto c_{\Omega, A, q, B f}^{*}(e) / \sqrt{B}$ remains, with the assumption $\nu$. A $\tilde{e}=0$ on $\partial \Omega$, bounded by two positive constants as $B$ gets very large. Therefore, it is interesting to find the limit of $c_{\Omega, A, q, B f}^{*}(e) / \sqrt{B}$ as $B \rightarrow+\infty$ even in some particular situations. Moreover, it is important to find the limit of the same quantity as $B \rightarrow 0^{+}$. We start with the case where $B \rightarrow+\infty$ and then we move to that where $B \rightarrow 0^{+}$.

Theorem 5.1 Let $e=(1,0, \ldots, 0) \in \mathbb{R}^{N}$ and $B>0$. Assume that $\Omega=\mathbb{R} \times \omega \subseteq \mathbb{R}^{N}, A$, and $f$ satisfy the same assumptions of Theorem 3.1. That is, $f$ and $A$ satisfy (3.5), (3.4), and (3.5), and one of the two alternatives (3.7)-(3.8). Consider the reaction-diffusion equation

$$
\left\{\begin{aligned}
u_{t}(t, x, y) & =\nabla \cdot(A(y) \nabla u)(t, x, y)+B f(x, y, u), \text { for }(t, x, y) \in \mathbb{R} \times \Omega, \\
\nu \cdot A \nabla u & =0 \text { on } \mathbb{R} \times \mathbb{R} \times \partial \omega
\end{aligned}\right.
$$

Then,

$$
\lim _{B \rightarrow+\infty} \frac{c_{\Omega, A, 0, B f}^{*}(e)}{\sqrt{B}}=2 \sqrt{\max _{y \in \bar{\omega}} \zeta(y)} \sqrt{\max _{y \in \bar{\omega}} e A e(y)} .
$$

We mention that one can find the coefficients $A$, and $f$ and the domain $\Omega$ of the problem (5.1) satisfying all the assumptions of Theorem 5.1, which are the same of Theorem 3.1, including one of the alternatives (3.7)-(3.8) while one of $\zeta$ and $e A e$ is not constant. Owing to Theorem 1.10 in [3], it follows that

$$
\forall B>0, \quad c_{\Omega, A, 0, B f}^{*}(e) \varsubsetneqq 2 \sqrt{B} \sqrt{\max _{y \in \bar{\omega}} \zeta(y)} \sqrt{\max _{y \in \bar{\omega}} e A e(y)},
$$

which is equivalent to saying that

$$
\frac{c_{\Omega, A, 0, B f}^{*}(e)}{\sqrt{B}} \varsubsetneqq 2 \sqrt{\max _{y \in \bar{\omega}} \zeta(y)} \sqrt{\max _{y \in \bar{\omega}} e A e(y)} .
$$

Therefore, there are heterogeneous settings in which the result found in Theorem 5.1 does not follow trivially.

We move now to study the limit when the reaction factor $B$ tends to $0^{+}$. However, the situation will be more general than that in Theorem 5.1 because it will consider reaction-advection-diffusion equations rather than considering reaction-diffusion equations only:

Theorem 5.2 Under the assumptions (2.1) for $\Omega$, (2.5) for the advection $q$, (2.4) and (2.5) for the nonlinearity $f=f(x, y, u)$, let $e$ be any unit direction of $\mathbb{R}^{d}$. Assume that the diffusion matrix $A=A(x, y)$ satisfies (2.8) together with $\nabla \cdot A \tilde{e} \equiv 0$ over $\Omega$, and $\nu \cdot A \tilde{e}=0$ over $\partial \Omega$. For each $B>0$ and $\gamma \geq 1 / 2$, consider the following reaction-advection-diffusion equation

$$
\left\{\begin{array}{l}
u_{t}=\nabla \cdot(A(x, y) \nabla u)+B^{\gamma} q(x, y) \cdot \nabla u+B f(x, y, u), \quad t \in \mathbb{R},(x, y) \in \Omega, \\
\nu \cdot A \nabla u(t, x, y)=0, \quad t \in \mathbb{R}, \quad(x, y) \in \partial \Omega .
\end{array}\right.
$$


Then

$$
\lim _{B \rightarrow 0^{+}} \frac{c_{\Omega, A, B^{\gamma} q, B f^{(e)}}^{*}}{\sqrt{B}}=2 \sqrt{f_{C} \tilde{e} A \tilde{e}(x, y) d x d y} \sqrt{f_{C} \zeta(x, y) d x d y},
$$

where $C$ is the periodicity cell of $\Omega$.

Having the above result one can mark a sample of notes:

The order of $B$ in the denominator of the ratio $c_{\Omega, A, B^{\gamma}}^{*}, B f(e) / \sqrt{B}$ is independent of $\gamma$ (it is equal to $1 / 2$ ). Thus, whenever the advection is null, one gets

$$
\lim _{B \rightarrow 0^{+}} \frac{c_{\Omega, A, 0, B f}^{*}(e)}{\sqrt{B}}=2 \sqrt{f_{C} \tilde{e} A \tilde{e}(x, y) d x d y} \sqrt{f_{C} \zeta(x, y) d x d y} .
$$

Therefore, one concludes that the presence of an advection with a factor $B^{\gamma}$, where $\gamma \geq 1 / 2$, will have no more effect on the limit of the ratio $c_{\Omega, A}^{*} B^{\gamma} q, B f(e) / \sqrt{B}$ as the reaction factor $B$ gets very small.

On the other hand, it is easy to check that the assumptions in Theorem 5.2 are more general than those in Theorem 5.1. Consequently, once we are in the more strict setting, which is that of Theorem 5.1, we are able to know both limits of $c_{\Omega, A, 0, B f}^{*}(e) / \sqrt{B}$ as $B \rightarrow+\infty$ and as $B \rightarrow 0^{+}$.

\section{Variations of the minimal speed with respect to diffusion and reaction factors and with respect to periodicity parameters}

After having studied the limits and the asymptotic behaviors of the of the functions $\varepsilon \mapsto c_{\Omega, \varepsilon A, 0, f}^{*}(e) / \sqrt{\varepsilon}$, $M \mapsto c_{\Omega, M A}^{*} M^{\gamma} q, f^{(e) / \sqrt{M}}$ (for very large $M$ and for $\left.0 \leq \gamma \leq 1 / 2\right), B \mapsto c_{\Omega, A, B^{\gamma} q, B f^{(e)} / \sqrt{B}}^{*}$ $(\gamma \geq 1 / 2)$ and $L \mapsto c_{\mathbb{R}^{N}, A_{L}, q_{L}, f_{L}}^{*}(e)$, where $L$ is a periodicity parameter, we move now to investigate the variations of these functions with respect to the diffusion and reaction factors and with respect the periodicity parameter $L$. The present section will be devoted to discuss and answer these questions.

We sketch first the form of the domain. $\Omega \subseteq \mathbb{R}^{N}$ is assumed to be in the form $\mathbb{R} \times \omega$ which was taken in section 3. As a review, $\Omega=\mathbb{R} \times \omega \subseteq \mathbb{R}^{N}$, where $\omega \subseteq \mathbb{R}^{d} \times \mathbb{R}^{N-d-1}(d \geq 0)$. If $d=0$, the subset $\omega$ is a bounded open subset of $\mathbb{R}^{N-1}$. While, in the case where $1 \leq d \leq N-1, \omega$ is a $\left(L_{1}, \ldots, L_{d}\right)$-periodic open domain of $\mathbb{R}^{N-1}$ which satisfies $(2.1)$; and hence, $\Omega$ is a $\left(l, L_{1}, \ldots, L_{d}\right)-$ periodic subset of $\mathbb{R}^{N}$ that satisfies (2.1) with $l>0$. An element of $\Omega=\mathbb{R} \times \omega$ will be represented as $z=(x, y)$ where $y \in \omega \subseteq \mathbb{R}^{d} \times \mathbb{R}^{N-1-d}$. With a domain of such form, we have:

Theorem 6.1 Let $e=(1,0, \ldots, 0) \in \mathbb{R}^{N}$. Assume that $\Omega$ has the form $\mathbb{R} \times \omega$ which is described above, and that the diffusion matrix $A=A(y)$ satisfies (3.5) together with the assumption

$$
A(x, y) e=A(y) e=\alpha(y) e, \text { for all }(x, y) \in \mathbb{R} \times \bar{\omega} ;
$$

where $y \mapsto \alpha(y)$ is a positive $\left(L_{1}, \ldots, L_{d}\right)$ - periodic function defined over $\bar{\omega}$. The nonlinearity $f$ is assumed to satisfy (3.3) and (3.4). Moreover, one assumes that, at least, one of $\tilde{e} \cdot$ A $\tilde{e}$ and $\zeta$ is not constant. Besides, the advection field $q$ (when it exists) is in the form $q(x, y)=\left(q_{1}(y), 0, \ldots, 0\right)$ 
where $q_{1}$ has a zero average over $C$, the periodicity cell of $\omega$. For each $\beta>0$ consider the reactionadvection-diffusion problem

$$
\left\{\begin{array}{l}
u_{t}=\beta \nabla \cdot(A(y) \nabla u)+\sqrt{\beta} q_{1}(y) \partial_{x} u+f(x, y, u), t \in \mathbb{R},(x, y) \in \mathbb{R} \times \omega \\
\nu \cdot A \nabla u(t, x, y)=0, \quad t \in \mathbb{R},(x, y) \in \partial \Omega
\end{array}\right.
$$

Then the map $\beta \mapsto \frac{c_{\Omega, \beta A, \sqrt{\beta} q, f}^{*}(e)}{\sqrt{\beta}}$ is decreasing in $\beta>0$, and by Theorem 4.1, one has

$$
\lim _{\beta \rightarrow+\infty} \frac{c_{\Omega, \beta A, \sqrt{\beta} q, f}^{*}(e)}{\sqrt{\beta}}=2 \sqrt{f_{C} \tilde{e} A \tilde{e}(y) d y} \sqrt{f_{C} \zeta(y) d y},
$$

where $C$ is the periodicity cell of $\omega$.

Remark 6.2 In the same setting of Theorem 6.1 but with no advection, that is $q_{1} \equiv 0$, we still have $\beta \mapsto \frac{c_{\Omega, \beta A, 0, f}^{*}(e)}{\sqrt{\beta}}$ as a decreasing map in $\beta>0$. Moreover, if one of the alternatives (3.7)-(3.8) holds and there is no advection, Theorem 3.1 yields that

$$
\lim _{\beta \rightarrow 0^{+}} \frac{c_{\Omega, \beta A, 0, f}^{*}(e)}{\sqrt{\beta}}=2 \sqrt{\max _{y \in \bar{\omega}} \tilde{e} A \tilde{e}(y)} \sqrt{\max _{y \in \bar{\omega}} \zeta(y)} .
$$

The preceding result yields another one concerned in the variation of the minimal speeds with respect to the periodicity parameter $L$. In the following, the domain will be the whole space $\mathbb{R}^{N}$. We choose the diffusion matrix $A(x, y)=A(y)$, the shear flow $q$ and reaction term $f$ to be $(1, \ldots, 1)$-periodic and to satisfy some restrictions. For each $L>0$, we assign the diffusion matrix $A_{L}(x, y)=A\left(\frac{x}{L}, \frac{y}{L}\right)$, the advection field $q_{L}(x, y)=q\left(\frac{x}{L}, \frac{y}{L}\right)$ and the nonlinearity $f_{L}=f\left(\frac{x}{L}, \frac{y}{L}, u\right)$ and we are going to study the variation, with respect to the periodicity parameter $L$, of the minimal speed $c_{\mathbb{R}^{N}, A_{L}, q_{L}, f_{L}}^{*}(e)$, which corresponds to the reaction-advection-diffusion equation within the $(L, \cdots, L)$-periodic coefficients $A_{L}, q_{L}$ and $f_{L}$ :

Theorem 6.3 Let $e=(1,0, \ldots, 0) \in \mathbb{R}^{N}$. An element $z \in \mathbb{R}^{N}$ is represented as $z=(x, y) \in \mathbb{R} \times$ $\mathbb{R}^{N-1}$. Assume that $A(x, y)=A(y)$ (for all $(x, y) \in \mathbb{R}^{N}$ ) and $f(x, y, u)$ satisfy ( (3.3), (3.4) and 3.5) with $\omega=\mathbb{R}^{N-1}, d=N-1$, and $l=L_{1}=\ldots=L_{N-1}=1$. Assume furthermore, that for all $y \in \mathbb{R}^{N-1}, A(x, y) e=A(y) e=\alpha(y)$ e, where $y \mapsto \alpha(y)$ is a positive $(1, \ldots, 1)$-periodic function defined over $\mathbb{R}^{N-1}$ and that, at least, one of $\tilde{e} \cdot A \tilde{e}$ and $\zeta$ is not constant. Let $q$ be an advection field satisfying (2.3) and having the form $q(x, y)=\left(q_{1}(y), 0 \ldots, 0\right)$ for each $(x, y) \in \mathbb{R}^{N}$. Consider the reaction-advection-diffusion problem,

$$
\begin{gathered}
\forall(t, x, y) \in \mathbb{R} \times \mathbb{R}^{N}, \\
u_{t}(t, x, y)=\nabla \cdot\left(A_{L}(y) \nabla u\right)(t, x, y)+\left(q_{1}\right)_{L}(y) \partial_{x} u(t, x, y)+f_{L}(x, y, u),
\end{gathered}
$$

whose coefficients are $(L, \ldots, L)$-periodic with respect to $(x, y) \in \mathbb{R}^{N}$.

Then, the map $L \mapsto c_{\mathbb{R}^{N}, A_{L}, q_{L}, f_{L}}^{*}(e)$ is increasing in $L>0$. 
Remark 6.4 The assumptions of Theorem 6.3 can not be fulfilled whenever $N=1$. However, assuming that $N=1$ and that the function

$$
\frac{\zeta}{<\zeta>_{A}}+\frac{<a>_{H}}{a}
$$

is not identically equal to 2 (where $a(x)$ is the diffusion factor, $\langle a\rangle_{H}$ and $\langle\zeta\rangle_{A}$ are, respectively, the harmonic mean of $x \mapsto a(x)$ and arithmetic mean of $x \mapsto \zeta(x)$ over $[0,1])$, it was proved, in [13], that $L \mapsto c_{\mathbb{R}^{N}, a_{L}, q_{L}, f_{L}}^{*}(e)$ is increasing in $L$ when $L$ is close to 0 . In particular, if a is constant and $\zeta$ is not constant, or if $\mu$ is constant and a is not constant, then $L \mapsto c_{\mathbb{R}^{N}, a_{L}, q_{L}, f_{L}}(e)$ is increasing when $L$ is close to 0.

Concerning now the variation with respect to the reaction factor $B$, we have the following:

Theorem 6.5 Assume that $\Omega=\mathbb{R} \times \omega$ and the coefficients $A, q$ and $f$ satisfy the same assumptions of Theorem 6.1. Let $e=(1,0 \ldots, 0)$ and for each $B>0$, consider the reaction-advection-diffusion problem

$$
\left\{\begin{array}{l}
u_{t}=\nabla \cdot(A(y) \nabla u)+\sqrt{B} q_{1}(y) \partial_{x} u+B f(x, y, u), \quad t \in \mathbb{R},(x, y) \in \mathbb{R} \times \omega \\
\nu \cdot A \nabla u(t, x, y)=0, \quad t \in \mathbb{R},(x, y) \in \partial \Omega
\end{array}\right.
$$

Then, the map $B \mapsto \frac{c_{\Omega, A, \sqrt{B} q, B f}^{*}(e)}{\sqrt{B}}$ is increasing in $B>0$.

As a first note, we mention that Theorem 6.5 holds also in the case where there is no advection. On the other hand, Berestycki, Hamel and Nadirashvili [3] proved that the map $B \mapsto c_{\Omega, A, q, B f}^{*}(e)$ is increasing in $B>0$ under the assumptions (2.1), (2.2), (2.3), (2.4), and (2.5) which are less strict than the assumptions considered in our present theorem. However, the present theorem is concerned in the variation of the map $B \mapsto \frac{c_{\Omega, A, \sqrt{B} q, B f}^{*}(e)}{\sqrt{B}}$ rather than that of $B \mapsto c_{\Omega, A, q, B f}^{*}(e)$.

Remark 6.6 Owing to the same justifications given after Theorem 3.5, one concludes the importance of taking, in section $\mathbb{G}$, an advection in the form of shear flows. To study the variations of the minimal speeds as in Theorems 6.1, 6.3 and 6.5, but in a more general framework (general advection fields, general diffusion, etc...), formula 2.7 remains an important tool. However, we will no longer have variational formulations as (7.69) below. These problems remains open in the general periodic framework.

\section{Proofs of the announced results}

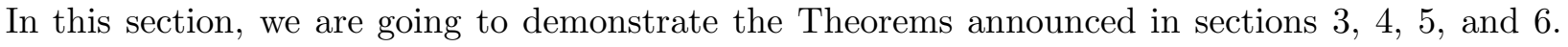
We will proceed in 4 subsections, each devoted to proving the results announced in a corresponding section. 


\subsection{Proofs of Theorems 3.1, 3.3 and 3.4}

Proof of Theorem 3.1. Under the assumptions of Theorem 3.1, we can apply the variational formula (2.7) of the minimal speed. Consequently,

$$
c_{\Omega, \varepsilon A, 0, f}^{*}(e)=\min _{\lambda>0} \frac{k_{\Omega, e, \varepsilon A, 0, \zeta}(\lambda)}{\lambda},
$$

where $k_{\Omega, e, \varepsilon A, 0, \zeta}(\lambda)$ is the first eigenvalue (for each $\lambda, \varepsilon>0$ ) of the eigenvalue problem

$$
\begin{cases}L_{\Omega, e, \varepsilon A, 0, \zeta, \lambda \psi} & =k_{\Omega, e}, \varepsilon A, 0, \zeta(\lambda) \psi(x, y) \text { over } \mathbb{R} \times \omega ; \\ \nu \cdot A \nabla \psi & =0 \quad \text { on } \mathbb{R} \times \partial \omega\end{cases}
$$

and

$$
\begin{aligned}
L_{\Omega, e, \varepsilon A, 0, \zeta, \lambda} \psi(x, y)= & \varepsilon \nabla \cdot(A(y) \nabla \psi(x, y))-2 \varepsilon \lambda A e \cdot \nabla \psi(x, y)+ \\
& {\left[\varepsilon \lambda^{2} e A(y) e-\lambda \varepsilon \nabla \cdot(A(y) e)+\zeta(y)\right] \psi(x, y), }
\end{aligned}
$$

for all $(x, y) \in \mathbb{R} \times \omega$.

Initially, the boundary condition in (7.2) is $\nu \cdot A \nabla \psi=\lambda \nu \cdot A e$ on $\partial \Omega=\mathbb{R} \times \partial \omega$; where $\nu(x, y)$ is the unit outward normal at $(x, y) \in \partial \Omega$. However, $\Omega=\mathbb{R} \times \omega$ is invariant in the direction of $e$ which is that of $A e$ in both alternatives (3.7) and (3.8). Consequently, $\nu \cdot A e \equiv 0$ on $\partial \Omega$.

We recall that for all $\lambda>0$, and for all $\varepsilon>0$, we have $k_{\Omega, e, \varepsilon A, 0, \zeta}(\lambda)>0$. Also, the first eigenfunction of (7.2) is positive over $\bar{\Omega}=\mathbb{R} \times \bar{\omega}$, and it is unique up to multiplication by a non zero constant.

In our present setting, whether in (3.7) or (3.8) and due to the assumption (3.4), one concludes that the coefficients in $L_{\Omega, e} \varepsilon A, 0, \zeta, \lambda$ are independent of $x$. Moreover, in both alternatives (3.7) and (3.8), the direction of $A e$ is the same of $e=(1,0, \cdots, 0)$. On the other hand, since $\Omega=\mathbb{R} \times \omega$, then for each $(x, y) \in \partial \Omega$, we have $\nu(x, y)=\left(0 ; \nu_{\omega}(y)\right)$, where $\nu_{\omega}(y)$ is the outward unit normal on $\partial \omega$ at $y$. Consequently, the first eigenfunction of (7.2) is independent of $x$ and the eigenvalue problem (7.2) is reduced to

$$
\left\{\begin{aligned}
L_{\Omega, e, \varepsilon A, 0, \zeta, \lambda^{\phi}:} & =\varepsilon \nabla \cdot(A(y) \nabla \phi(y))+\left[\varepsilon \lambda^{2} e A(y) e+\zeta(y)\right] \phi(y) \\
& =k_{\Omega, e}, \varepsilon A, 0, \zeta(\lambda) \phi \text { over } \omega ; \\
\nu(x, y) \cdot A(y) \nabla \phi(y) & =\left(0 ; \nu_{\omega}(y)\right) \cdot A(y) \nabla \phi(y)=0 \quad \text { on } \mathbb{R} \times \partial \omega
\end{aligned}\right.
$$

where $\phi=\phi(y)$ is positive over $\bar{\omega}, L$-periodic (since the domain $\omega$ and the coefficients of $L_{\Omega, e, \varepsilon A, 0, \zeta, \lambda}$ are $L$-periodic), unique up to multiplication by a constant, and belongs to $C^{2}(\bar{\omega})$.

In the case where $d \geq 1$, let $C \subseteq \mathbb{R}^{N-1}$ denote the periodicity cell of $\omega$. Otherwise, $d=0$ and one takes $C=\omega$. In both cases, $C$ is bounded. Multiplying the first line of (7.3) by $\phi$, and integrating by parts over $C$, one gets

$$
-k_{\Omega, e, \varepsilon A, 0, \zeta}(\lambda)=\frac{\varepsilon \int_{C} \nabla \phi \cdot A(y) \nabla \phi d y-\int_{C}\left[\varepsilon \lambda^{2} e A(y) e+\zeta(y)\right] \phi^{2}(y) d y}{\int_{C} \phi^{2}(y) d y} .
$$


One also notes that, in this present setting, the operator $L_{\Omega, e, \varepsilon A, 0, \zeta, \lambda}$ is self-adjoint and its coefficients are $\left(L_{1}, \ldots, L_{d}\right)$-periodic with respect $\left(y_{1}, \ldots, y_{d}\right)$. Consequently, $-k_{\Omega, e, \varepsilon} A, 0, \zeta(\lambda)$ has the following variational characterization:

$$
-k_{\Omega, e, \varepsilon A, 0, \zeta}(\lambda)=\min _{\varphi \in H^{1}(C) \backslash\{0\}} \frac{\varepsilon \int_{C} \nabla \varphi \cdot A(y) \nabla \varphi d y-\int_{C}\left[\varepsilon \lambda^{2} e A(y) e+\zeta(y)\right] \varphi^{2}(y) d y}{\int_{C} \varphi^{2}(y) d y} .
$$

In what follows, we will assume that (3.7) is the alternative that holds. That is, $e A e=\alpha$ is constant. The proof can be imitated easily whenever we assume that (3.8) holds.

The function $y \mapsto \zeta(y)$ is continuous and $\left(L_{1}, \ldots, L_{d}\right)$-periodic over $\bar{\omega}$, whose periodicity cell $C$ is a bounded subset of $\mathbb{R}^{N-1}$ (whether $d=0$ or $d \geq 1$ ). Let $y_{0} \in \bar{C} \subseteq \bar{\omega}$ such that $\max _{y \in \bar{w}} \zeta(y)=\zeta\left(y_{0}\right)$ (trivially, this also holds when $\zeta$ is constant). Consequently, we have

$$
\forall \varphi \in H^{1}(C) \backslash\{0\}, \frac{\varepsilon \int_{C} \nabla \varphi \cdot A \nabla \varphi-\int_{C}\left(\varepsilon \alpha \lambda^{2}+\zeta(y)\right) \varphi^{2}}{\int_{C} \varphi^{2}(y) d y} \geq-\left[\varepsilon \alpha \lambda^{2}+\zeta\left(y_{0}\right)\right] .
$$

This yields that

$$
\forall \varepsilon>0, \forall \lambda>0,-k_{\Omega, e, \varepsilon A, 0, \zeta}(\lambda) \geq-\left[\varepsilon \alpha \lambda^{2}+\zeta\left(y_{0}\right)\right]
$$

Consequently,

$$
\forall \varepsilon>0, \forall \lambda>0, \frac{k_{\Omega, e, \varepsilon A, 0, \zeta}(\lambda)}{\lambda} \leq \lambda \alpha \varepsilon+\frac{\zeta\left(y_{0}\right)}{\lambda}
$$

However, the function $\lambda \mapsto \lambda \alpha \varepsilon+\frac{\zeta\left(y_{0}\right)}{\lambda}$ attains its minimum, over $\mathbb{R}^{+}$, at $\lambda(\varepsilon)=\sqrt{\frac{\zeta\left(y_{0}\right)}{\alpha \varepsilon}}$. This minimum is equal to $2 \sqrt{\zeta\left(y_{0}\right)} \times \sqrt{\alpha \varepsilon}$. From (7.7), we conclude that

$$
\frac{k_{\Omega, e, \varepsilon A, 0, \zeta}(\lambda(\varepsilon))}{\lambda(\varepsilon)} \leq 2 \sqrt{\alpha \varepsilon} \sqrt{\zeta\left(y_{0}\right)} .
$$

Finally, 2.7) implies that $c_{\Omega, \varepsilon A, 0, f}^{*}(e)=\min _{\lambda>0} \frac{k_{\Omega, e, \varepsilon A, 0, \zeta}(\lambda)}{\lambda} \leq 2 \sqrt{\alpha \varepsilon} \sqrt{\zeta\left(y_{0}\right)}$, or equivalently

$$
\forall \varepsilon>0, \frac{c_{\Omega, \varepsilon A, 0, f}^{*}(e)}{\sqrt{\varepsilon}} \leq 2 \sqrt{\alpha} \sqrt{\zeta\left(y_{0}\right)}
$$

We pass now to prove the other sense of the inequality for $\liminf _{\varepsilon \rightarrow 0^{+}} \frac{c_{\Omega, \varepsilon A, 0, f}^{*}(e)}{\sqrt{\varepsilon}}$. We will consider formula (7.5), and then organize a suitable function $\psi$ which leads us to a lower bound of $\liminf _{\varepsilon \rightarrow 0^{+}} \frac{c_{\Omega, \varepsilon A, 0, f}^{*}(e)}{\sqrt{\varepsilon}}$.

We have $\zeta\left(y_{0}\right)>0$. Let $\delta$ be such that $0<\delta<\zeta\left(y_{0}\right)$. Thus $0<\zeta\left(y_{0}\right)-\delta<\max _{\bar{\omega}} \zeta(y)$. The continuity of $\zeta$, over $\bar{C} \subseteq \bar{\omega}$, yields that there exists an open and bounded set $U \subset \overline{\bar{C}}$ such that

$$
\forall y \in \bar{U}, \zeta\left(y_{0}\right)-\delta \leq \zeta(y) .
$$


Designate by $\psi$, a function in $\mathcal{D}(C)$ (a $C^{\infty}(C)$ function whose support is compact), with $\operatorname{supp} \psi \subseteq \bar{U}$, and $\int_{U} \psi^{2}=1$. One will have,

$$
\begin{aligned}
\forall \lambda>0, \forall \varepsilon>0, & \\
-k_{\Omega, e, \varepsilon A, 0, \zeta(\lambda)} & \leq \varepsilon \int_{U} \nabla \psi \cdot A(y) \nabla \psi d y-\int_{U}\left[\varepsilon \lambda^{2} e A(y) e+\zeta(y)\right] \psi^{2}(y) d y \\
& \leq \varepsilon \int_{U} \nabla \psi \cdot A(y) \nabla \psi d y-\left[\varepsilon \lambda^{2} \alpha+\zeta\left(y_{0}\right)-\delta\right] \int_{U} \psi^{2}(y) d y \\
& \leq \varepsilon \int_{U} \alpha_{2}|\nabla \psi|^{2}-\left[\varepsilon \lambda^{2} \alpha+\zeta\left(y_{0}\right)-\delta\right], \text { by (3.5), }
\end{aligned}
$$

or equivalently

$$
\frac{k_{\Omega, e, \varepsilon A, 0, \zeta}(\lambda)}{\lambda} \geq \lambda \alpha \varepsilon+\frac{1}{\lambda} \beta(\varepsilon)
$$

where $\beta(\varepsilon)=\zeta\left(y_{0}\right)-\delta-\varepsilon \int_{U} \alpha_{2}|\nabla \psi|^{2}$. Choosing $0<\varepsilon<\frac{\zeta\left(y_{0}\right)-\delta}{\alpha_{2} \int_{U}|\nabla \psi|^{2}}$ (this is possible), we get $\beta(\varepsilon)>0$.

The map $\lambda \mapsto \lambda \alpha \varepsilon+\frac{1}{\lambda} \beta(\varepsilon)$ attains its minimum, over $\mathbb{R}^{+}$, at $\lambda(\varepsilon)=\sqrt{\frac{\beta(\varepsilon)}{\varepsilon \alpha}}$. This minimum is equal to $2 \sqrt{\varepsilon \alpha} \sqrt{\beta(\varepsilon)}$.

Now, referring to formula (7.10), one gets

$$
\text { For } \varepsilon \text { small enough, } \quad \frac{k_{\Omega, e, \varepsilon A, 0, \zeta}(\lambda)}{\lambda} \geq 2 \sqrt{\varepsilon \alpha} \sqrt{\beta(\varepsilon)} \quad \text { for all } \lambda>0 .
$$

Together with (2.7), we conclude that

$$
\text { for } \varepsilon \text { small enough, } \frac{c_{\Omega, \varepsilon A, 0, f}^{*}(e)}{\sqrt{\varepsilon}} \geq 2 \sqrt{\beta(\varepsilon)} \sqrt{\alpha} \text {. }
$$

Consequently,

$$
\begin{aligned}
\liminf _{\varepsilon \rightarrow 0^{+}} \frac{c_{\Omega, \varepsilon A, 0, f}^{*}(e)}{\sqrt{\varepsilon}} & \geq \liminf _{\varepsilon \rightarrow 0^{+}} 2 \sqrt{\beta(\varepsilon)} \sqrt{\alpha} \\
& =2 \sqrt{\zeta\left(y_{0}\right)-\delta} \sqrt{\alpha} \quad(\text { since } \psi \text { is independent of } \varepsilon)
\end{aligned}
$$

and this holds for all $0<\delta<\zeta\left(y_{0}\right)$. Therefore, one can conclude that

$$
\liminf _{\varepsilon \rightarrow 0^{+}} \frac{c_{\Omega, \varepsilon A, 0, f}^{*}(e)}{\sqrt{\varepsilon}} \geq 2 \sqrt{\alpha} \sqrt{\zeta\left(y_{0}\right)}
$$

Finally, the inequalities (7.8) and (7.12) imply that $\lim _{\varepsilon \rightarrow 0^{+}} \frac{c_{\Omega, \varepsilon A, 0, f}^{*}(e)}{\sqrt{\varepsilon}}$ exists, and it is equal to

$$
2 \sqrt{\alpha} \sqrt{\zeta\left(y_{0}\right)}=2 \sqrt{\max _{\bar{\omega}} e A(y) e} \sqrt{\max _{\bar{\omega}} \zeta(y)}
$$


We note that the same ideas of this proof can be easily applied in the case where the assumption (3.8) holds. In (3.8), we have $\zeta$ is constant; however, $e A e$ is not in general. Meanwhile the converse is true in the case (3.7). The little difference is that, in the case of (3.8), we choose the subset $U$ (of the proof done above) around the point $y_{0}$ where $e A e$ attains its maximum and then we continue by the same way used above.

Proof of Theorem 3.3. We have

$$
c_{\Omega, \varepsilon A, 0, f}^{*}(e)=\min _{\lambda>0} \frac{k_{\Omega, e, \varepsilon A, q, \zeta}(\lambda)}{\lambda},
$$

where (due to the facts that $q$ is a shear flow, $e=(1,0, \cdots, 0)$ and $e$ is an eigenvector of the matrix $A(y)$ for all $y \in \bar{\omega}) k_{\Omega, e, \varepsilon A, q, \zeta}(\lambda)$ is the principal eigenvalue of the problem

$$
\begin{cases}L_{\Omega, e, \varepsilon A, q, \zeta, \lambda} \psi(x, y) & =k_{\Omega, e, \varepsilon A, q, \zeta}(\lambda) \psi(x, y) \quad \text { over } \mathbb{R} \times \omega ; \\ \nu \cdot A \nabla \psi & =0 \text { on } \mathbb{R} \times \partial \omega\end{cases}
$$

with

$$
\begin{aligned}
L_{\Omega, e, \varepsilon A, q, \zeta, \lambda} \psi= & \varepsilon \nabla \cdot(A(y) \nabla \psi)-2 \varepsilon \lambda \alpha(y) \partial_{x} \psi+q_{1}(y) \partial_{x} \psi \\
& +\left[\varepsilon \lambda^{2} e A(y) e-\lambda q_{1}(y)+\zeta(y)\right] \psi \text { over } \mathbb{R} \times \omega .
\end{aligned}
$$

The uniqueness of the principal eigenfunction $\psi$ up to multiplication by a constant, yields that one can choose $\psi$ independent of $x$. Hence, the elliptic operator $L_{\Omega, e, \varepsilon A, q, \zeta, \lambda}$ can be reduced to the symmetric operator

$$
L_{\Omega, e, \varepsilon A, q, \zeta, \lambda} \psi=\varepsilon \nabla \cdot(A(y) \nabla \psi)+\left[\varepsilon \lambda^{2} e A(y) e-\lambda q_{1}(y)+\zeta(y)\right] \psi .
$$

Consequently,

$$
\begin{aligned}
& \forall \lambda>0, \forall \varepsilon>0, \quad-k_{\Omega, e, \varepsilon A, q, \zeta}(\lambda)= \\
& \min _{\varphi \in H^{1}(C) \backslash\{0\}} \frac{\varepsilon \int_{C} \nabla \varphi \cdot A(y) \nabla \varphi d y+\lambda \int_{C} q_{1}(y) \varphi^{2}-\int_{C}\left[\lambda^{2} \varepsilon e A(y) e+\zeta(y)\right] \varphi^{2}(y) d y}{\int_{C} \varphi^{2}(y) d y} .
\end{aligned}
$$

Formula (7.15) yields that

$$
\forall \lambda>0, \forall \varepsilon>0,-k_{\Omega, e, \varepsilon A, q, \zeta}(\lambda) \geq-\lambda \max _{y \in \bar{\omega}}\left(-q_{1}(y)\right)-\lambda^{2} \varepsilon \max _{y \in \bar{\omega}} e A(y) e-\max _{y \in \bar{\omega}} \zeta(y),
$$

or equivalently

$$
\forall \lambda>0, \forall \varepsilon>0, \frac{k_{\Omega, e, \varepsilon A, q, \zeta}(\lambda)}{\lambda} \leq \max _{y \in \bar{\omega}}\left(-q_{1}(y)\right)+\lambda \varepsilon \max _{y \in \bar{\omega}} e A(y) e+\frac{\max _{y \in \bar{\omega}} \zeta(y)}{\lambda} .
$$

Putting $\lambda=\lambda(\varepsilon)=\sqrt{\frac{\max _{y \in \bar{\omega}} \zeta(y)}{\varepsilon \max _{y \in \bar{\omega} e \cdot A(y) e}}}>0$ into the last inequality yields that

$$
\min _{\lambda>0} \frac{k_{\Omega, e, \varepsilon A, q, \zeta^{(\lambda)}}}{\lambda} \leq \max _{y \in \bar{\omega}}\left(-q_{1}(y)\right)+2 \sqrt{\varepsilon} \sqrt{\max _{y \in \bar{\omega}} e \cdot A(y) e} \sqrt{\max _{y \in \bar{\omega}} \zeta(y)},
$$


and hence,

$$
\limsup _{\varepsilon \rightarrow 0^{+}} c_{\Omega, \varepsilon A, q, f}^{*}(e) \leq \max _{y \in \bar{\omega}}\left(-q_{1}(y)\right) .
$$

Now, we take $y_{0} \in C(C$ is the periodicity cell of $\omega)$ such that $\max _{y \in \bar{\omega}}\left(-q_{1}(y)\right)=-q_{1}\left(y_{0}\right)>0$ (since $q$ is periodic with respect to $y, q_{1} \not \equiv 0$ and $q_{1}$ has a zero average) and we take $\delta>0$ such $-q_{1}\left(y_{0}\right)-\delta>0$. It follows, from the continuity of $q_{1}$, that there exists an open subset $U \subset C$ such that $y_{0} \in U$ and

$$
\forall y \in \bar{U},-q_{1}(y) \geq \max _{y \in \bar{\omega}}\left(-q_{1}(y)\right)-\delta .
$$

Let $\psi$ be a function in $\mathcal{D}(C)$ with $\operatorname{supp} \psi \subseteq \bar{U}$, and $\int_{U} \psi^{2}=1$. Referring to ( that

$$
\forall \lambda>0, \forall \varepsilon>0, \quad \frac{k_{\Omega, e, \varepsilon A, q, \zeta}(\lambda)}{\lambda} \geq-q_{1}\left(y_{0}\right)-\delta+\lambda \varepsilon \min _{y \in \bar{\omega}} e \cdot A e+\frac{1}{\lambda} \beta(\varepsilon),
$$

where $\beta(\varepsilon)=\min _{y \in \bar{\omega}} \zeta(y)-\varepsilon \int_{U} \alpha_{2}|\nabla \psi|^{2}>0$ for a small enough $\varepsilon>0\left(\alpha_{2}>0\right.$ is the constant appearing in (3.5)).

It follows from $(7.17)$ that

$$
\forall \lambda>0, \forall \varepsilon>0, \quad \frac{k_{\Omega, e}, \varepsilon A, q, \zeta^{(\lambda)}}{\lambda} \geq-q_{1}\left(y_{0}\right)-\delta+2 \sqrt{\varepsilon} \sqrt{\min _{y \in \bar{\omega}} e \cdot A e} \sqrt{\beta(\varepsilon)} .
$$

Together with (7.13), and since $\delta>0$ is arbitrary, one gets

$$
\liminf _{\varepsilon \rightarrow 0^{+}} c_{\Omega, \varepsilon A, q, f}^{*}(e) \geq-q_{1}\left(y_{0}\right)=\max _{y \in \bar{\omega}}\left(-q_{1}(y)\right) .
$$

Finally, (7.16) and (7.18) complete the proof of Theorem 3.3.

Proof of Theorem 3.4. Consider the change of variables

$$
v(t, x, y)=u(t, L x, L y), \quad(t, x, y) \in \mathbb{R} \times \mathbb{R} \times \mathbb{R}^{N-1} .
$$

The function $u$ satisfies (3.12) if and only if $v$ satisfies

$$
v_{t}(t, x, y)=\frac{1}{L^{2}} \nabla \cdot(A(y) \nabla v)(t, x, y)+f(x, y, v) \text { over } \mathbb{R} \times \mathbb{R} \times \mathbb{R}^{N-1} .
$$

Consequently,

$$
\forall L>0, c_{\mathbb{R}^{N}}^{*}, A_{L}, 0, f_{L}(e)=L c_{\mathbb{R}^{N}, \frac{1}{L^{2}} A, 0, f}^{*}(e)
$$

Taking $\varepsilon=1 / L^{2}$, and applying Theorem 3.1 to problem (7.19), one then has

$$
\lim _{L \rightarrow+\infty} \frac{c_{\mathbb{R}^{N}, \frac{1}{L^{2}} A, 0, f}^{*}(e)}{\sqrt{\frac{1}{L^{2}}}}=\lim _{\varepsilon \rightarrow 0^{+}} \frac{c_{\mathbb{R}^{N}, \varepsilon A, 0, f}^{*}(e)}{\sqrt{\varepsilon}}=2 \sqrt{\max _{y \in \mathbb{R}^{N-1}} \zeta(y)} \sqrt{\max _{y \in \mathbb{R}^{N-1}} e A(y) e} .
$$

Finally, (7.20) together with (7.21) complete the proof of Theorem 3.4. 


\subsection{Proofs of Theorems 4.1 and 4.3}

Proof of Theorem 4.1. The proof will be divided into three steps:

Step 1. According to Theorem 2.4, and since $\nu \cdot A \tilde{e}=0$ on $\partial \Omega$, the minimal speeds $c_{\Omega, M A, M^{\gamma} q, f}^{(e)}$ are given by:

$$
\forall M>0, c_{\Omega, M A, M^{\gamma}}^{*} q, f(e)=\min _{\lambda>0} \frac{k_{\Omega, e}, M A, M^{\gamma} q, \zeta^{(\lambda)}}{\lambda},
$$

where $k_{\Omega, e}, M A, M^{\gamma} q, \zeta(\lambda)$ and $\psi^{\lambda, M}$ denote the unique eigenvalue and the positive $L$-periodic eigenfunction of the problem

$$
\begin{gathered}
M \nabla \cdot\left(A \nabla \psi^{\lambda, M}\right)-2 M \lambda \tilde{e} \cdot A \nabla \psi^{\lambda, M}+M^{\gamma} q \cdot \nabla \psi^{\lambda, M}+\left[\lambda^{2} M \tilde{e} A \tilde{e}-\lambda M^{\gamma} q \cdot \tilde{e}+\zeta\right] \psi^{\lambda, M} \\
=k_{\Omega, e,} M A, M^{\gamma} q, \zeta(\lambda) \psi^{\lambda, M} \text { in } \Omega
\end{gathered}
$$

with $\nu \cdot A \nabla \psi^{\lambda, M}=0$ on $\partial \Omega$.

For each $\lambda>0$ and $M>0$, let $\lambda^{\prime}=\lambda \sqrt{M}$, and let $k_{\Omega, e, M A, M^{\gamma} q, \zeta}(\lambda)=\mu\left(\lambda^{\prime}, M\right)$. Consequently,

$$
\forall M>0, \frac{c_{\Omega, M A}^{*}, M^{\gamma} q, f^{(e)}}{\sqrt{M}}=\min _{\lambda^{\prime}>0} \frac{\mu\left(\lambda^{\prime}, M\right)}{\lambda^{\prime}},
$$

where $\mu\left(\lambda^{\prime}, M\right)$ and $\psi^{\lambda^{\prime}, M}$ are the first eigenvalue and the unique, positive $L$-periodic (with respect to $x$ ) eigenfunction of

$$
\begin{gathered}
M \nabla \cdot\left(A \nabla \psi^{\lambda^{\prime}, M}\right)-2 \lambda^{\prime} \sqrt{M} \tilde{e} \cdot A \nabla \psi^{\lambda^{\prime}, M}+M^{\gamma} q \cdot \nabla \psi^{\lambda^{\prime}, M} \\
+\left[\lambda^{\prime 2} \tilde{e} A \tilde{e}-\frac{\lambda^{\prime}}{M^{\frac{1}{2}-\gamma}} q \cdot \tilde{e}+\zeta\right] \psi^{\lambda^{\prime}, M}=\mu\left(\lambda^{\prime}, M\right) \psi^{\lambda^{\prime}, M} \text { in } \Omega,
\end{gathered}
$$

with $\nu \cdot A \nabla \psi^{\lambda^{\prime}, M}=0$ on $\partial \Omega$.

Owing to the uniqueness, up to multiplication by positive constants, of the first eigenfunction of (7.23), one may assume that:

$$
\forall \lambda^{\prime}>0, \forall M>0,\left\|\psi^{\lambda^{\prime}, M}\right\|_{L^{2}(C)}=1
$$

Moreover, for each $M>0, \min _{\lambda^{\prime}>0} \frac{\mu\left(\lambda^{\prime}, M\right)}{\lambda^{\prime}}$ is attained at $\lambda_{M}^{\prime}>0$. Thus,

$$
\forall M>0, \frac{c_{\Omega, M A, M^{\gamma} q, f^{(e)}}}{\sqrt{M}}=\min _{\lambda^{\prime}>0} \frac{\mu\left(\lambda^{\prime}, M\right)}{\lambda^{\prime}}=\frac{\mu\left(\lambda_{M}^{\prime}, M\right)}{\lambda_{M}^{\prime}} .
$$

The above characterization of $c_{\Omega, M A}^{*} M^{\gamma} q, f(e) / \sqrt{M}$ will be used in the next steps in order to prove that $\liminf _{M \rightarrow+\infty} c_{\Omega, M A, M^{\gamma}}^{*} q, f^{(e) / \sqrt{M}}\left(\operatorname{resp} . \limsup _{M \rightarrow+\infty} c_{\Omega, M A}^{*} M^{\gamma} q, f^{(e) / \sqrt{M}}\right)$ is greater than (resp. less than) $2 \sqrt{f_{C} \tilde{e} A \tilde{e}(x, y) d x d y} \sqrt{f_{C} \zeta(x, y) d x d y}$; and hence, complete the proof. 
Step 2. Fix $\lambda^{\prime}>0$ and $M>0$. We divide $(7.23)$ by $\psi^{\lambda^{\prime}, M}$ then, using the facts $\nabla . A \tilde{e} \equiv 0$ in $\Omega$ and $\overline{\nu \cdot A \tilde{e}}=0$ on $\partial \Omega$, we integrate by parts over the periodicity cell $C$. It follows from (2.3) and the $L$-periodicity of $A, \zeta$ and $\psi^{\lambda^{\prime}, M}$ that

$$
\int_{C} \frac{\nabla \psi^{\lambda^{\prime}, M} \cdot A \nabla \psi^{\lambda^{\prime}, M}}{\left(\psi^{\lambda^{\prime}, M}\right)^{2}}+\lambda^{\prime 2} \int_{C} \tilde{e} A \tilde{e}+\int_{C} \zeta=\mu\left(\lambda^{\prime}, M\right)|C|,
$$

where $|C|$ denotes the Lebesgue measure of $C$. Let

$$
m_{0}=f_{C} \tilde{e} A \tilde{e}=\frac{1}{|C|} \int_{C} \tilde{e} A(x, y) \tilde{e} d x d y \quad \text { and } \quad m=f_{C} \zeta(x, y) d x d y .
$$

One concludes that

$$
\forall \lambda^{\prime}>0, \forall M>0, \quad \mu\left(\lambda^{\prime}, M\right) \geq \lambda^{\prime 2} f_{C} \tilde{e} A \tilde{e}+f_{C} \zeta=\lambda^{\prime 2} m_{0}+m,
$$

whence

$$
\forall \lambda^{\prime}>0, \forall M>0, \quad \frac{\mu\left(\lambda^{\prime}, M\right)}{\lambda^{\prime}} \geq \lambda^{\prime} m_{0}+\frac{m}{\lambda^{\prime}}
$$

The right side of $(\sqrt{7.27})$ attains its minimum over $\mathbb{R}^{+}$at $\lambda_{0}^{\prime}=\sqrt{\frac{m}{m_{0}}}$. This minimum is equal to $2 \sqrt{m_{0} m}$.

Consequently, for any $M>0, \frac{c_{\Omega, M A, M^{\gamma} q, f}^{*}(e)}{\sqrt{M}}=\min _{\lambda^{\prime}>0} \frac{\mu\left(\lambda^{\prime}, M\right)}{\lambda^{\prime}} \geq 2 \sqrt{m_{0} m}$. This yields that

$$
\liminf _{M \rightarrow+\infty} \frac{c_{\Omega, M A, M^{\gamma} q, f^{(e)}}^{*}}{\sqrt{M}} \geq 2 \sqrt{f_{C} \tilde{e} A \tilde{e}(x, y) d x d y} \sqrt{f_{C} \zeta(x, y) d x d y} .
$$

Step 3. Fix $\lambda^{\prime}>0$ and $M>0$. Multiply (7.23) by $\psi^{\lambda^{\prime}, M}$ and integrate by parts over $C$. Owing to the $L$-periodicity of $\Omega, A, \zeta$ and $\psi^{\lambda^{\prime}, M}$, and due to the facts that $\int_{C}\left(\psi^{\lambda^{\prime}, M}\right)^{2}=1, \nabla \cdot A \tilde{e} \equiv 0$ in $\Omega$, and that $\nu \cdot A \tilde{e}=0$ on $\partial \Omega$, together with (2.3), one gets

$$
\begin{gathered}
-M \int_{C} \nabla \psi^{\lambda^{\prime}, M} \cdot A \nabla \psi^{\lambda^{\prime}, M}+\lambda^{\prime 2} \int_{C} \tilde{e} A \tilde{e}\left(\psi^{\lambda^{\prime}, M}\right)^{2}+\int_{C} \zeta\left(\psi^{\lambda^{\prime}, M}\right)^{2} \\
-\frac{\lambda^{\prime}}{M^{\frac{1}{2}-\gamma}} \int_{C} q \cdot \tilde{e}\left(\psi^{\lambda^{\prime}, M}\right)^{2}=\mu\left(\lambda^{\prime}, M\right),
\end{gathered}
$$

whence

$$
\forall \lambda^{\prime}>0, \forall M>0,0<\mu\left(\lambda^{\prime}, M\right) \leq \lambda^{\prime 2} \alpha+\beta+\frac{\lambda^{\prime}}{M^{\frac{1}{2}-\gamma}}\left\|(q \cdot \tilde{e})^{-}\right\|_{\infty},
$$

where $\alpha=\max _{(x, y) \in \bar{\Omega}} \tilde{e} A \tilde{e}(x, y)$ and $\beta=\max _{(x, y) \in \bar{\Omega}} \zeta(x, y)$. Together with $(7.27)$, one gets

$$
\forall \lambda^{\prime}>0, \forall M>0,0<\lambda^{\prime 2} m_{0}+m \leq \mu\left(\lambda^{\prime}, M\right) \leq \lambda^{\prime 2} \alpha+\beta+\frac{\lambda^{\prime}}{M^{\frac{1}{2}-\gamma}}\left\|(q \cdot \tilde{e})^{-}\right\|_{\infty} .
$$


If $\gamma=\frac{1}{2}$, then $\frac{\lambda^{\prime}}{M^{\frac{1}{2}-\gamma}}\left\|(q \cdot \tilde{e})^{-}\right\|_{\infty}=\lambda^{\prime}\left\|(q \cdot \tilde{e})^{-}\right\|_{\infty}$. On the other hand, if $0 \leq \gamma<\frac{1}{2}$, then

$$
\frac{\lambda^{\prime}}{M^{\frac{1}{2}-\gamma}}\left\|(q \cdot \tilde{e})^{-}\right\|_{\infty} \rightarrow 0 \quad \text { as } \quad M \rightarrow+\infty .
$$

Consequently, the right side of $(7.30)$ is bounded above by a positive constant $B$ which does not depend on $M$ and $\gamma$. This yields that

$$
\forall \lambda^{\prime}>0, \quad 0<\limsup _{M \rightarrow+\infty} \mu\left(\lambda^{\prime}, M\right)<+\infty .
$$

On the other hand, it follows from (2.2) and (7.29) that $\forall \lambda^{\prime}>0, \forall M>0$,

$$
\begin{aligned}
0 & \leq \alpha_{1} \int_{C}\left|\nabla \psi^{\lambda^{\prime}, M}\right|^{2} \leq \int_{C} \nabla \psi^{\lambda^{\prime}, M} \cdot A \nabla \psi^{\lambda^{\prime}, M} \\
& \leq \frac{1}{M}\left[-\mu\left(\lambda^{\prime}, M\right)+\lambda^{\prime 2} \int_{C} \tilde{e} A \tilde{e}\left(\psi^{\lambda^{\prime}, M}\right)^{2}+\int_{C} \zeta\left(\psi^{\lambda^{\prime}, M}\right)^{2}-\frac{\lambda^{\prime}}{M^{\frac{1}{2}-\gamma}} \int_{C} q \cdot \tilde{e}\left(\psi^{\lambda^{\prime}, M}\right)^{2}\right] \\
& <\frac{B}{M} .
\end{aligned}
$$

Meanwhile, $\lim _{M \rightarrow+\infty} \frac{B}{M}=0$, one then gets

$$
\left\{\begin{array}{l}
\forall \lambda^{\prime}>0, \quad \lim _{M \rightarrow+\infty} \int_{C}\left|\nabla \psi^{\lambda^{\prime}, M}\right|^{2}=0, \\
\forall \lambda^{\prime}>0, \forall M>0, \quad \int_{C}\left(\psi^{\lambda^{\prime}, M}\right)^{2}=1 .
\end{array}\right.
$$

Fix $\lambda^{\prime}>0$, and let $\left(M_{n}\right)_{n}$ be a sequence converging to $+\infty$ as $n \rightarrow+\infty$ and such that $\mu\left(\lambda^{\prime}, M_{n}\right) \rightarrow l^{\lambda^{\prime},\left(M_{n}\right)}$ as $n \rightarrow+\infty$. It follows, from (7.31), that $\left\|\psi^{\lambda^{\prime}, M_{n}}\right\|_{H^{1}(C)} \rightarrow 1$ as $n \rightarrow+\infty$. Thus, the sequence $\left(\psi^{\lambda^{\prime}, M_{n}}\right)_{n}$ is bounded in $H^{1}(C)$. Therefore, there exists a function $\psi^{\lambda^{\prime}, \infty} \in$ $H^{1}(C)$ such that, up to extraction of some subsequence, the functions $\left(\psi^{\lambda^{\prime}, M_{n}}\right)_{n}$ converge in $L^{2}(C)$ strong, $H^{1}(C)$ weak and almost everywhere in $C$, to the function $\psi^{\lambda^{\prime}}, \infty$. Consequently, and owing to (7.31), $\psi^{\lambda^{\prime}, \infty}$ satisfies

$$
\begin{gathered}
\int_{C}\left(\psi^{\lambda^{\prime}, \infty}\right)^{2}=1, \text { and } \\
\left(\int_{C}\left|\nabla \psi^{\lambda^{\prime}, \infty}\right|^{2}\right)^{\frac{1}{2}} \leq \liminf _{M_{n} \rightarrow+\infty}\left(\int_{C}\left|\nabla \psi^{\lambda^{\prime}, M_{n}}\right|^{2}\right)^{\frac{1}{2}}=0 .
\end{gathered}
$$

From (7.33), it follows that for all $\lambda^{\prime}>0$, the function $\psi^{\lambda^{\prime}, \infty}$ is almost everywhere constant over $C$. On the other hand, the elliptic regularity applied on equation $(\overline{7.23})$ for $M=M_{n}$, implies that $\forall \lambda^{\prime}>0$, the function $\psi^{\lambda^{\prime}, \infty}$ is continuous over $\bar{C}$. Consequently, referring to (7.32), one gets

$$
\forall \lambda^{\prime}>0, \quad \psi^{\lambda^{\prime}, \infty}=\frac{1}{\sqrt{|C|}} \text { over } \bar{C} .
$$


Consider now equation (7.23). Fix $\lambda^{\prime}$, take $M=M_{n}$, and integrate by parts over $C$. It follows, from (2.2), (2.3) and the assumptions $\nabla . A \tilde{e} \equiv 0$ over $\Omega$ with $\nu$.A $=0$ on $\partial \Omega$, that $\int_{C} M_{n} \nabla \cdot\left(A \nabla \psi^{\lambda^{\prime}, M_{n}}\right)=0, \int_{C}-2 \lambda^{\prime} \sqrt{M_{n}} \tilde{e} \cdot A \nabla \psi^{\lambda^{\prime}, M_{n}}=0$, and $\int_{C} q \cdot \nabla \psi^{\lambda^{\prime}, M_{n}}=0$. Hence,

$$
-\frac{\lambda^{\prime}}{M_{n}^{\frac{1}{2}-\gamma}} \int_{C} q \cdot \tilde{e} \psi^{\lambda^{\prime}, M_{n}}+\lambda^{\prime 2} \int_{C} \tilde{e} \cdot A \tilde{e} \psi^{\lambda^{\prime}, M_{n}}+\int_{C} \zeta \psi^{\lambda^{\prime}, M_{n}}=\mu\left(\lambda^{\prime}, M_{n}\right) \int_{C} \psi^{\lambda^{\prime}, M_{n}} .
$$

Meanwhile, the functions $\psi^{\lambda^{\prime}, M_{n}}$ converge to the constant function $\psi^{\lambda^{\prime}, \infty}$ in $L^{2}(C)$ strong; and hence, in $L^{1}(C)$ strong ( $C$ is bounded, so $L^{2}(C)$ is embedded in $L^{1}(C)$ ). Let $M_{n} \rightarrow+\infty$ in (7.35):

In case $\gamma=1 / 2$, one has

$$
\frac{\lambda^{\prime}}{M_{n}^{\frac{1}{2}-\gamma}} \int_{C} q \cdot \tilde{e} \psi^{\lambda^{\prime}, M_{n}}=\lambda^{\prime} \int_{C} q \cdot \tilde{e} \psi^{\lambda^{\prime}, M_{n}} \rightarrow \lambda^{\prime} \psi^{\lambda^{\prime}, \infty} \int_{C} q \cdot \tilde{e}=0,
$$

as $n \rightarrow+\infty$ (from $(2.3))$. Also, in the case $0 \leq \gamma<1 / 2$, one trivially has

$$
\frac{\lambda^{\prime}}{M_{n}^{\frac{1}{2}-\gamma}} \int_{C} q \cdot \tilde{e} \psi^{\lambda^{\prime}, M_{n}} \rightarrow 0 \text { as } n \rightarrow+\infty
$$

Moreover, $\tilde{e} A \tilde{e}$ and $\zeta$ are in $L^{\infty}(C)$. Thus, as $M_{n} \rightarrow+\infty$ in (7.35), we get

$$
\lambda^{\prime 2} \psi^{\lambda^{\prime}, \infty} \int_{C} \tilde{e} A \tilde{e}+\psi^{\lambda^{\prime}, \infty} \int_{C} \zeta=l^{\lambda^{\prime},\left(M_{n}\right)} \psi^{\lambda^{\prime}, \infty}|C| .
$$

One concludes that

$$
\forall \lambda^{\prime}>0, \quad \frac{l^{\lambda^{\prime},\left(M_{n}\right)}}{\lambda^{\prime}}=\lambda^{\prime} f_{C} \tilde{e} A \tilde{e}+\frac{f_{C} \zeta}{\lambda^{\prime}}=\lambda^{\prime} m_{0}+\frac{m}{\lambda^{\prime}} .
$$

Whence for $\lambda^{\prime}=\lambda_{0}^{\prime}=\sqrt{\frac{m}{m_{0}}}$, one gets $\frac{l^{\lambda_{0}^{\prime},\left(M_{n}\right)}}{\lambda_{0}^{\prime}}=2 \sqrt{m_{0} m}$.

On the other hand, for all $M_{n}$,

$$
\frac{c_{\Omega, M_{n} A, M_{n}^{\gamma} q, f^{(e)}}^{*}}{\sqrt{M_{n}}}=\inf _{\lambda^{\prime}>0} \frac{\mu\left(\lambda^{\prime}, M_{n}\right)}{\lambda^{\prime}} \leq \frac{\mu\left(\lambda_{0}^{\prime}, M_{n}\right)}{\lambda_{0}^{\prime}} .
$$

Passing $M_{n} \rightarrow+\infty$, one gets $\limsup _{M_{n} \rightarrow+\infty} \frac{c_{\Omega, M_{n} A, M_{n}^{\gamma} q, f}^{*}(e)}{\sqrt{M_{n}}} \leq \frac{l^{\lambda_{0}^{\prime},\left(M_{n}\right)}}{\lambda_{0}^{\prime}}=2 \sqrt{m_{0} m}$, and this holds for all sequences $\left\{M_{n}\right\}_{n}$ converging to $+\infty$. Thus,

$$
\limsup _{M \rightarrow+\infty} \frac{c_{\Omega, M A, M^{\gamma} q, f^{(e)}}^{*}}{\sqrt{M}} \leq 2 \sqrt{f_{C} \tilde{e} A \tilde{e}(x, y) d x d y} \sqrt{f_{C} \zeta(x, y) d x d y}
$$

Having (7.28) together with (7.38), the proof of Theorem 4.1 is complete. 
Proof of Theorem 4.3. We will consider the change of variables similar to that made in the proof of Theorem 3.4:

$$
v(t, x, y)=u(t, L x, L y), \quad(t, x, y) \in \mathbb{R} \times \mathbb{R}^{N} .
$$

After the same calculations done there, one gets that $u$ satisfies (4.1) if and only if $v$ satisfies

$$
v_{t}(t, x, y)=\frac{1}{L^{2}} \nabla \cdot(A(x, y) \nabla v)(t, x, y)+\frac{1}{L} q \cdot \nabla v(t, x, y)+f(x, y, v) \text { over } \mathbb{R} \times \mathbb{R}^{N} .
$$

Consequently,

$$
\forall L>0, c_{\mathbb{R}^{N}}^{*}, A_{L}, q_{L}, f_{L}(e)=L c_{\mathbb{R}^{N}, \frac{1}{L^{2}} A, \frac{1}{L} q, f}^{*}(e) .
$$

On the other hand, the coefficients and the domain of problem (7.39) satisfy all the assumptions of Theorem 4.1. Taking $M=1 / L^{2}$ and $\gamma=1 / 2$, then (7.39) can be rewritten as

$$
v_{t}(t, x, y)=M \nabla \cdot(A(x, y) \nabla v)(t, x, y)+M^{\frac{1}{2}} q \cdot \nabla v(t, x, y)+f(x, y, v) \text { over } \mathbb{R} \times \mathbb{R}^{N} .
$$

In this situation, the periodicity cell of the whole space $\mathbb{R}^{N}$ is $C=[0,1] \times \cdots \times[0,1]$.

It follows, from Theorem 4.1, that

$$
\begin{aligned}
\lim _{L \rightarrow 0^{+}} \frac{c_{\mathbb{R}^{N}, \frac{1}{L^{2}} A, \frac{1}{L} q, f}(e)}{\sqrt{\frac{1}{L^{2}}}} & =\lim _{M \rightarrow+\infty} \frac{c_{\mathbb{R}^{N}, M A, M^{\frac{1}{2}} q, f}^{*}}{\sqrt{M}} \\
& =2 \sqrt{f_{C} \tilde{e} A \tilde{e}(x, y) d x d y} \sqrt{f_{C} \zeta(x, y) d x d y}
\end{aligned}
$$

Having (7.40) together with (7.41), the proof of Theorem 4.3 is complete.

\subsection{Proofs of Theorems 5.1 and 5.2}

Proof of Theorem 5.1. The main ideas of this proof are similar to those in the demonstration of Theorem 3.1. Applying the variational formula (2.7) of the minimal speed, one gets

$$
c_{\Omega, A, 0, B f}^{*}(e)=\min _{\lambda>0} \frac{k_{\Omega, e, A, 0, B \zeta}(\lambda)}{\lambda},
$$

where $k_{\Omega, e, A, 0, B \zeta}(\lambda)$ is the first eigenvalue (for each $\lambda, B>0$ ) of the eigenvalue problem:

$$
\begin{cases}L_{\Omega, e, A, 0, B \zeta, \lambda} \psi(x, y) & =k_{\Omega, e, A, 0, B \zeta}(\lambda) \psi(x, y) \text { over } \mathbb{R} \times \omega \\ \nu \cdot A \nabla \psi & =0 \quad \text { on } \mathbb{R} \times \partial \omega\end{cases}
$$

and

$$
\begin{aligned}
L_{\Omega, e, A, 0, B \zeta, \lambda} \psi(x, y)= & \nabla \cdot(A(y) \nabla \psi(x, y))-2 \lambda A e \cdot \nabla \psi(x, y)+ \\
& {\left[\lambda^{2} e A(y) e-\lambda \nabla \cdot(A(y) e)+B \zeta(y)\right] \psi(x, y), }
\end{aligned}
$$

for each $(x, y) \in \mathbb{R} \times \omega$. 
We recall that for all $\lambda>0$, and for all $B>0$, we have $k_{\Omega, e, A, 0, B \zeta}(\lambda)>0$. Also, the first eigenfunction of (7.43) is positive over $\bar{\Omega}=\mathbb{R} \times \bar{\omega}$, and it is unique up to multiplication by a non zero constant.

Moreover, whether in (3.7) or (3.8) and due to (3.4), one concludes that the coefficients in $L_{\Omega, e, A, 0, B \zeta, \lambda}$ are independent of $x$. Hence, the first eigenfunction of (7.43) is independent of $x$ and the eigenvalue problem (7.43) is reduced to

$$
\left\{\begin{aligned}
L_{\Omega, e, A, 0, B \zeta, \lambda} \phi & :=\nabla \cdot(A(y) \nabla \phi(y))+\left[\lambda^{2} e A(y) e+B \zeta(y)\right] \phi(y) \\
& =k_{\Omega, e, A, 0, B \zeta}(\lambda) \phi \text { over } \omega ; \\
\nu(x, y) \cdot A(y) \nabla \phi(y) & =\left(0 ; \nu_{\omega}(y)\right) \cdot A(y) \nabla \phi(y)=0 \quad \text { on } \mathbb{R} \times \partial \omega
\end{aligned}\right.
$$

where $\phi=\phi(y)$ is positive over $\bar{\omega}, L$-periodic (since the domain $\omega$ and the coefficients of $L_{\Omega, e, A, 0, B \zeta, \lambda}$ are $L$-periodic), unique up to multiplication by a constant, and belongs to $C^{2}(\bar{\omega})$.

In the case where $d \geq 1$, let $C \subseteq \mathbb{R}^{N-1}$ denote the periodicity cell of $\omega$. Otherwise, $d=0$ and one takes $C=\omega$. In both cases, $C$ is bounded. Multiplying the first line of (7.44) by $\phi$, and integrating by parts over $C$, one gets

$$
-k_{\Omega, e, A, 0, B \zeta}(\lambda)=\frac{\int_{C} \nabla \phi \cdot A(y) \nabla \phi d y-\int_{C}\left[\lambda^{2} e A(y) e+B \zeta(y)\right] \phi^{2}(y) d y}{\int_{C} \phi^{2}(y) d y} .
$$

One also notes that, in this present setting, the operator $L_{\Omega, e, A, 0, B \zeta, \lambda}$ is self-adjoint and its coefficients are $\left(L_{1}, \ldots, L_{d}\right)$-periodic with respect $\left(y_{1}, \ldots, y_{d}\right)$. Consequently, $-k_{\Omega, e, A, 0, B \zeta}(\lambda)$ has the following variational characterization:

$$
-k_{\Omega, e, A, 0, B \zeta}(\lambda)=\min _{\varphi \in H^{1}(C) \backslash\{0\}} \frac{\int_{C} \nabla \varphi \cdot A(y) \nabla \varphi d y-\int_{C}\left[\lambda^{2} e A(y) e+B \zeta(y)\right] \varphi^{2}(y) d y}{\int_{C} \varphi^{2}(y) d y} .
$$

In what follows, we will assume that (3.7) is the alternative that holds. That is, $e A e=\alpha$ is constant. The proof can be imitated easily whenever we assume that (3.8) holds.

The function $y \mapsto \zeta(y)$ is continuous and $\left(L_{1}, \ldots, L_{d}\right)$-periodic over $\bar{\omega}$, whose periodicity cell $C$ is a bounded subset of $\mathbb{R}^{N-1}$ (whether $d=0$ or $d \geq 1$ ). Let $y_{0} \in \bar{C} \subseteq \bar{\omega}$ such that $\max _{y \in \bar{w}} \zeta(y)=\zeta\left(y_{0}\right)$ (trivially, this also holds when $\zeta$ is constant). Consequently, we have

$$
\forall \varphi \in H^{1}(C) \backslash\{0\}, \frac{\int_{C} \nabla \varphi \cdot A \nabla \varphi-\int_{C}\left(\alpha \lambda^{2}+B \zeta(y)\right) \varphi^{2}}{\int_{C} \varphi^{2}(y) d y} \geq-\left[\alpha \lambda^{2}+B \zeta\left(y_{0}\right)\right] .
$$

This yields that

$$
\forall B>0, \forall \lambda>0,-k_{\Omega, e, A, 0, B \zeta}(\lambda) \geq-\left[\alpha \lambda^{2}+B \zeta\left(y_{0}\right)\right]
$$

Consequently,

$$
\forall B>0, \forall \lambda>0, \frac{k_{\Omega, e, A, 0, B \zeta}(\lambda)}{\lambda} \leq \lambda \alpha+\frac{B \zeta\left(y_{0}\right)}{\lambda} .
$$


However, the function $\lambda \mapsto \lambda \alpha+\left(B \zeta\left(y_{0}\right) / \lambda\right)$ attains its minimum, over $\mathbb{R}^{+}$, at $\lambda(B)=\sqrt{\frac{B \zeta\left(y_{0}\right)}{\alpha}}$. This minimum is equal to $2 \sqrt{B \zeta\left(y_{0}\right)} \times \sqrt{\alpha}$.

From (7.48), we conclude that: $\frac{k_{\Omega, e, A, 0, B \zeta}(\lambda(B))}{\lambda(B)} \leq 2 \sqrt{B \alpha} \sqrt{\zeta\left(y_{0}\right)}$.

Finally, (2.7) implies that

$$
c_{\Omega, A, 0, B f}^{*}(e)=\min _{\lambda>0} \frac{k_{\Omega, e, A, 0, B \zeta}(\lambda)}{\lambda} \leq 2 \sqrt{B \alpha} \sqrt{\zeta\left(y_{0}\right)},
$$

or equivalently

$$
\forall B>0, \frac{c_{\Omega, A, 0, B f}^{*}(e)}{\sqrt{B}} \leq 2 \sqrt{\alpha} \sqrt{\zeta\left(y_{0}\right)}
$$

We pass now to prove the other sense of the inequality for $\liminf _{B \rightarrow+\infty} \frac{c_{\Omega, A, 0, B f}^{*}(e)}{\sqrt{B}}$. We will consider formula (7.5), and then organize a suitable function $\psi$ which leads us to a lower bound of $\liminf _{B \rightarrow+\infty} \frac{c_{\Omega, A, 0, B f}^{*}(e)}{\sqrt{B}}$.

We have $\zeta\left(y_{0}\right)>0$. Let $\delta$ be such that $0<\delta<\zeta\left(y_{0}\right)$. Thus $0<\zeta\left(y_{0}\right)-\delta<\max _{\bar{\omega}} \zeta(y)$. The continuity of $\zeta$, over $\bar{C} \subseteq \bar{\omega}$, yields that there exists an open and bounded set $U \subset \overline{\bar{\omega}}$ such that

$$
\zeta\left(y_{0}\right)-\delta \leq \zeta(y), \forall y \in \bar{U}
$$

Designate by $\psi$, a function in $\mathcal{D}(C)$ (a $C^{\infty}(C)$ function whose support is compact), with $\operatorname{supp} \psi \subseteq \bar{U}$, and $\int_{U} \psi^{2}=1$. One will have,

$$
\begin{aligned}
\forall \lambda>0, \forall B>0, & \\
-k_{\Omega, e, A, 0, B \zeta}(\lambda) & \leq \int_{U} \nabla \psi \cdot A(y) \nabla \psi d y-\int_{U}\left[\lambda^{2} e A(y) e+B \zeta(y)\right] \psi^{2}(y) d y \\
& \leq \int_{U} \nabla \psi \cdot A(y) \nabla \psi d y-\left[\lambda^{2} \alpha+B\left(\zeta\left(y_{0}\right)-\delta\right)\right](\text { by (7.50) }) \\
& \left.\leq \int_{U} \alpha_{2}|\nabla \psi|^{2}-\left[\lambda^{2} \alpha+B\left(\zeta\left(y_{0}\right)-\delta\right)\right] \text { by (3.5) }\right)
\end{aligned}
$$

or equivalently

$$
\frac{k_{\Omega, e, A}, 0, B \zeta(\lambda)}{\lambda} \geq \lambda \alpha+\frac{B}{\lambda} \rho(B)
$$

where $\rho(B)=\zeta\left(y_{0}\right)-\delta-\frac{1}{B} \int_{U} \alpha_{2}|\nabla \psi|^{2}$. Choosing $B$ large enough, we get $\rho(B)>0$ (this is possible since $\zeta\left(y_{0}\right)-\delta>0$ and also $\left.\int_{U} \alpha_{2}|\nabla \psi|^{2}>0\right)$. The map $\lambda \mapsto \lambda \alpha+\frac{B}{\lambda} \rho(B)$ attains its minimum, over $\mathbb{R}^{+}$, at $\lambda(\varepsilon)=\sqrt{\frac{B \rho(B)}{\alpha}}$. This minimum is equal to $2 \sqrt{B \alpha} \sqrt{\rho(B)}$.

Now, referring to formula (7.51), one gets:

$$
\text { for } B \text { large enough, } \frac{k_{\Omega, e, A, 0, B \zeta(\lambda)}}{\lambda} \geq 2 \sqrt{B \alpha} \sqrt{\rho(B)} \text { for all } \lambda>0 \text {. }
$$


Together with (2.7), we conclude that

$$
\text { for } B \text { large enough, } \frac{c_{\Omega, A, 0, B f^{(e)}}^{*}}{\sqrt{B}} \geq 2 \sqrt{\rho(B)} \sqrt{\alpha} \text {. }
$$

Consequently,

$$
\begin{aligned}
\liminf _{B \rightarrow+\infty} \frac{c_{\Omega, A, 0, B f}^{*}(e)}{\sqrt{B}} & \geq \liminf _{B \rightarrow+\infty} 2 \sqrt{\rho(B)} \sqrt{\alpha} \\
& =2 \sqrt{\zeta\left(y_{0}\right)-\delta} \sqrt{\alpha} \quad(\text { since } \psi \text { is independent of } B),
\end{aligned}
$$

and this holds for all $0<\delta<\zeta\left(y_{0}\right)$. Therefore, one can conclude that

$$
\liminf _{B \rightarrow+\infty} \frac{c_{\Omega, A, 0, B f}^{*}(e)}{\sqrt{B}} \geq 2 \sqrt{\alpha} \sqrt{\zeta\left(y_{0}\right)}
$$

Finally, the inequalities $(7.49)$ and $(7.53)$ imply that $\lim _{B \rightarrow+\infty} \frac{c_{\Omega, A, 0, B f}^{*}(e)}{\sqrt{B}}$ exists, and it is equal to $2 \sqrt{\alpha} \sqrt{\zeta\left(y_{0}\right)}=2 \sqrt{\max _{\bar{\omega}} e A(y) e} \sqrt{\max _{\bar{\omega}} \zeta(y)}$.

The above proof was done while assuming that the alternative (3.7) holds. The same ideas of this proof can be easily applied in the case where alternative (3.8) holds. In (3.8), we have $\zeta$ is constant; however, eAe is not in general. Meanwhile the converse is true in the case (3.7). The little difference is that, in the case of (3.8), we chsose the subset $U$ (of the proof done above) around the point $y_{0}$ where $e A e$ attains its maximum and then we continue by the same way used above.

Proof of Theorem 5.2. According to Theorem 2.4, and since $\nu \cdot A \tilde{e}=0$ on $\partial \Omega$, the minimal speeds $c_{\Omega, A, B^{\gamma}}^{*}, B f^{(e)}$ are given by:

$$
\forall B>0, c_{\Omega, A, B}^{*} q, B f(e)=\min _{\lambda>0} \frac{k_{\Omega, e, A} B^{\gamma} q, B \zeta(\lambda)}{\lambda},
$$

where $k_{\Omega, e, A} B^{\gamma} q, B \zeta(\lambda)$ and $\psi^{\lambda, B}$ denote the unique eigenvalue and the positive $L$-periodic eigenfunction of the problem

$$
\begin{gathered}
\nabla \cdot\left(A \nabla \psi^{\lambda, B}\right)-2 \lambda \tilde{e} \cdot A \nabla \psi^{\lambda, B}+B^{\gamma} q \cdot \nabla \psi^{\lambda, B}+\left[\lambda^{2} \tilde{e} A \tilde{e}-\lambda B^{\gamma} q \cdot \tilde{e}+B \zeta\right] \psi^{\lambda, B} \\
\quad=k_{\Omega, e, A} B^{\gamma} q, B \zeta^{(\lambda) \psi^{\lambda, B}} \text { in } \Omega, \text { with } \nu \cdot A \nabla \psi=\nu \cdot A \nabla \psi^{\lambda, B}=0 \text { on } \partial \Omega .
\end{gathered}
$$

For each $\lambda>0$ and $B>0$, let $\lambda^{\prime}=\lambda / \sqrt{B}$, and let $k_{\Omega, e, A}, B^{\gamma} q, B \zeta(\lambda)=\mu\left(\lambda^{\prime}, B\right)$. Consequently,

$$
\forall B>0, \frac{c_{\Omega, A, B^{\gamma} q, B f^{(e)}}^{*}}{\sqrt{B}}=\min _{\lambda^{\prime}>0} \frac{\mu\left(\lambda^{\prime}, B\right)}{\lambda^{\prime} B},
$$

where $\mu\left(\lambda^{\prime}, B\right)$ and $\psi^{\lambda^{\prime}, B}$ are the first eigenvalue and the unique, positive $L$-periodic (with respect to $x$ ) eigenfunction of

$$
\begin{gathered}
\nabla \cdot\left(A \nabla \psi^{\lambda^{\prime}, B}\right)-2 \lambda^{\prime} \sqrt{B} \tilde{e} \cdot A \nabla \psi^{\lambda^{\prime}, B}+B^{\gamma} q \cdot \nabla \psi^{\lambda^{\prime}, B} \\
+\left[\lambda^{\prime 2} B \tilde{e} A \tilde{e}-\lambda^{\prime} B^{\gamma+\frac{1}{2}} q \cdot \tilde{e}+B \zeta\right] \psi^{\lambda^{\prime}, B}=\mu\left(\lambda^{\prime}, B\right) \psi^{\lambda^{\prime}, B} \text { in } \Omega
\end{gathered}
$$


with $\nu \cdot A \nabla \psi^{\lambda^{\prime}, B}=0$ on $\partial \Omega$.

Owing to the uniqueness, up to multiplication by positive constants, of the first eigenfunction of (7.55), one may assume that:

$$
\forall \lambda^{\prime}>0, \forall B>0,\left\|\psi^{\lambda^{\prime}, B}\right\|_{L^{2}(C)}=1
$$

Moreover, for each $B>0, \min _{\lambda^{\prime}>0} \frac{\mu\left(\lambda^{\prime}, B\right)}{\lambda^{\prime} B}$ is attained at $\lambda_{B}^{\prime}>0$. Thus,

$$
\forall B>0, \frac{c_{\Omega, A, B^{\gamma} q, B f^{(e)}}^{*}}{\sqrt{B}}=\min _{\lambda^{\prime}>0} \frac{\mu\left(\lambda^{\prime}, B\right)}{\lambda^{\prime} B}=\frac{\mu\left(\lambda_{B}^{\prime}, B\right)}{B \lambda_{B}^{\prime}} .
$$

Having the above characterization, one can now imitate the steps 2 and 3 in the proof of Theorem 4.1 to prove that

$$
\liminf _{B \rightarrow 0^{+}} c_{\Omega, A, B^{\gamma}}^{*}, B f^{(e) / \sqrt{B}}
$$

(resp. $\left.\limsup _{B \rightarrow 0^{+}} c_{\Omega, A}^{*} B^{\gamma} q, B f^{(e) / \sqrt{B}}\right)$ is greater than (resp. less than)

$$
2 \sqrt{f_{C} \tilde{e} A \tilde{e}(x, y) d x d y} \sqrt{f_{C} \zeta(x, y) d x d y}
$$

and hence, complete the proof of Theorem 5.2 .

\subsection{Proofs of Theorems 6.1, 6.3, and 6.5}

Proof of Theorem 6.1. Referring to Theorem 2.4, it follows that for each $\beta>0$, we have:

$$
\frac{c_{\Omega, \beta A, \sqrt{\beta} q, f}^{*}(e)}{\sqrt{\beta}}=\min _{\lambda>0} \frac{k_{\Omega, e, \beta A, \sqrt{\beta} q, \zeta}(\lambda)}{\lambda \sqrt{\beta}},
$$

where $k_{\Omega, e, \beta A, \sqrt{\beta} q, \zeta}(\lambda)$ is the first eigenvalue of the problem

$$
\left\{\begin{aligned}
L_{\Omega, e, \beta A, \sqrt{\beta} q, \zeta, \lambda} \psi(x, y) & =k_{\Omega, e, \beta A, \sqrt{\beta} q, \zeta}(\lambda) \psi(x, y) \text { over } \mathbb{R} \times \omega ; \\
\nu . A \nabla \psi & =0 \text { on } \mathbb{R} \times \partial \omega
\end{aligned}\right.
$$

where

$$
\begin{aligned}
L_{\Omega, e, \beta A, \sqrt{\beta} q, \zeta, \lambda} \psi= & \beta \nabla \cdot(A(y) \nabla \psi)-2 \beta \lambda \alpha(y) \partial_{x} \psi+\sqrt{\beta} q_{1}(y) \partial_{x} \psi \\
& +\left[\beta \lambda^{2} e A(y) e-\lambda \sqrt{\beta} q_{1}(y)+\zeta(y)\right] \psi \text { over } \mathbb{R} \times \omega .
\end{aligned}
$$

The boundary condition follows so from the facts that $\Omega=\mathbb{R} \times \omega, e=(1,0, \ldots, 0)$ and that $A(y) e=\alpha(y) e$ over $\omega$. These yield that $\nu \cdot A e=0$ over $\partial \Omega$ and $\nabla \cdot A e=0$. Moreover, for each $(x, y) \in \partial \Omega$, we have $\nu(x, y)=\left(0 ; \nu_{\omega}(y)\right)$, where $\nu_{\omega}(y)$ is the outward unit normal on $\partial \omega$ at $y$.

On the other hand, the function $\psi$ is positive,$\left(L_{1}, \ldots, L_{d}\right)$-periodic with respect to $y$, and unique up to multiplication by non-zero constants. Meanwhile, the coefficients $A, q$ and $\zeta$ are 
independent of $x$. Thus the eigenfunction $\psi$ will be independent of $x$ and our eigenvalue problem is reduced to

$$
\left\{\begin{array}{c}
\beta \nabla \cdot(A(y) \nabla \psi(y))+\left[\beta \lambda^{2} e A(y) e-\lambda \sqrt{\beta} q_{1}(y)+\zeta(y)\right] \psi(y) \\
=k_{\Omega, e}, \beta A, \sqrt{\beta} q, \zeta(\lambda) \psi(y) \text { for all } y \in \omega \\
\nu(x, y) \cdot A(y) \nabla \psi(y)=\left(0 ; \nu_{\omega}(y)\right) \cdot A(y) \nabla \psi(y)=0 \text { on } \mathbb{R} \times \partial \omega .
\end{array}\right.
$$

For each $\lambda>0$ and $\beta>0$, let $\lambda^{\prime}=\lambda \sqrt{\beta}$, and let $k_{\Omega, e, \beta A, \sqrt{\beta} q, \zeta}(\lambda)=\mu\left(\lambda^{\prime}, \beta\right)$. Since for each $\beta>0, \min _{\lambda>0} \frac{k_{\Omega, e, \beta A, \sqrt{\beta} q, \zeta}(\lambda)}{\lambda}$ is attained at $\lambda(\beta)$, it follows that

$$
\forall \beta>0, \quad \frac{c_{\Omega, \beta A, \sqrt{\beta} q, f}^{*}(e)}{\sqrt{\beta}}=\min _{\lambda^{\prime}>0} \frac{\mu\left(\lambda^{\prime}, \beta\right)}{\lambda^{\prime}},
$$

where $\mu\left(\lambda^{\prime}, \beta\right)$ is the first eigenvalue of the problem:

$$
\left\{\begin{aligned}
L_{\lambda^{\prime}}^{\beta} \psi & =\beta \nabla \cdot(A(y) \nabla \psi)+\left[\lambda^{\prime 2} e A(y) e-\lambda^{\prime} q_{1}(y)+\zeta(y)\right] \psi=\mu\left(\lambda^{\prime}, \beta\right) \psi \text { in } \omega, \\
\nu \cdot A \nabla \psi & =0 \text { on } \partial \omega .
\end{aligned}\right.
$$

The elliptic operator $L_{\lambda^{\prime}}^{\beta}$ in (7.61) is self-adjoint. Consequently, the first eigenvalue $\mu\left(\lambda^{\prime}, \beta\right)$ has the following characterization:?

$$
\begin{aligned}
\forall \lambda^{\prime}>0, \forall \beta>0, \quad-\mu\left(\lambda^{\prime}, \beta\right)= & \min _{\varphi \in H^{1}(C) \backslash\{0\}} \frac{\beta \int_{C} \nabla \varphi \cdot A(y) \nabla \varphi d y+\lambda^{\prime} \int_{C} q_{1}(y) \varphi^{2}-\int_{C}\left[\lambda^{\prime 2} e A(y) e+\zeta(y)\right] \varphi^{2}(y) d y}{\int_{C} \varphi^{2}(y) d y} \\
& =\min _{\varphi \in H^{1}(C) \backslash\{0\}} R\left(\lambda^{\prime}, \beta, \varphi\right) .
\end{aligned}
$$

For each $\lambda^{\prime}$ and $\beta>0, \varphi \mapsto R\left(\lambda^{\prime}, \beta, \varphi\right)$ attains its minimum over $H^{1}(C) \backslash\{0\}$ at $\psi^{\lambda^{\prime}, \beta}$, the eigenfunction of the problem (7.61). On the other hand, $\beta \mapsto R\left(\lambda^{\prime}, \beta, \varphi\right)$ is increasing as an affine function in $\beta$. Consequently, fixing $\lambda^{\prime}>0$ and taking $\beta>\beta^{\prime}>0$ :

$$
\begin{aligned}
-\mu\left(\lambda^{\prime}, \beta\right) & =R\left(\lambda^{\prime}, \beta, \psi^{\lambda^{\prime}, \beta}\right)>R\left(\lambda^{\prime}, \beta^{\prime}, \psi^{\lambda^{\prime}, \beta}\right) \\
& \geq \min _{\varphi \in H^{1}(C) \backslash\{0\}} R\left(\lambda^{\prime}, \beta^{\prime}, \varphi\right)=-\mu\left(\lambda^{\prime}, \beta^{\prime}\right) .
\end{aligned}
$$

In other words, for all $\lambda^{\prime}>0$, the function $\beta \mapsto \mu\left(\lambda^{\prime}, \beta\right)$ is decreasing. Concerning now the function $\beta \mapsto c_{\Omega, \beta A, \sqrt{\beta} q, f}^{*}(e) / \sqrt{\beta}$, one takes randomly $\beta>\beta^{\prime}>0$, hence

$$
\begin{aligned}
\frac{c_{\Omega, \beta^{\prime} A, \sqrt{\beta^{\prime}} q, f}^{*}(e)}{\sqrt{\beta^{\prime}}} & =\frac{\mu\left(\lambda^{\prime}\left(\beta^{\prime}\right), \beta^{\prime}\right)}{\lambda^{\prime}\left(\beta^{\prime}\right)}>\frac{\mu\left(\lambda^{\prime}\left(\beta^{\prime}\right), \beta\right)}{\lambda^{\prime}\left(\beta^{\prime}\right)} \\
& \geq \min _{\lambda^{\prime}>0} \frac{\mu\left(\lambda^{\prime}, \beta\right)}{\lambda^{\prime}}=\frac{c_{\Omega, \beta A, \sqrt{\beta} q, f}^{*}(e)}{\sqrt{\beta}},
\end{aligned}
$$

\footnotetext{
${ }^{2}$ To have an idea, multiply $(7.61)$ by the positive, $\left(L_{1}, \ldots, L_{d}\right)$-periodic function $\psi$ and integrate by parts over the periodicity cell $C$ of the the domain $\omega$.
} 
which means that the function $\beta \mapsto c_{\Omega, \beta A, \sqrt{\beta} q, f}^{*}(e) / \sqrt{\beta}$ is decreasing.

Finally, when $\beta \rightarrow+\infty$, one can easily check that the hypothesis of Theorem 4.1 are satisfied; hence, one has the limit at $+\infty$, and that completes the proof of Theorem 6.1.

Proof of Theorem 6.3. Consider the change of variables $v(t, x, y)=u(t, L x, L y)$, for any $(t, x, y) \in \mathbb{R} \times \mathbb{R}^{N}$. One consequently has,

$$
\forall L>0, c_{\mathbb{R}^{N}}^{*}, A_{L}, q_{L}, f_{L}(e)=L c_{\mathbb{R}^{N}, \frac{1}{L^{2}}}^{*} A, \frac{1}{L} q, f(e) .
$$

Taking $\beta=1 / L^{2}$, then

$$
v_{t}(t, x, y)=\beta \nabla \cdot(A(y) \nabla v)(t, x, y)+\sqrt{\beta} q_{1}(y) \partial_{x} v(t, x, y)+f(x, y, v) \text { over } \mathbb{R} \times \mathbb{R}^{N} .
$$

Owing to Theorem 6.1, the function $\beta \mapsto c_{\mathbb{R}^{N}, \beta A, \sqrt{\beta} q, f}^{(e) / \sqrt{\beta}}$ is decreasing in $\beta>0$. Besides, $L \mapsto 1 / L^{2}$ is decreasing in $L>0$. Together with (7.64), one obtains that the function $L \mapsto c_{\mathbb{R}^{N}}^{*}, A_{L}, q_{L}, f_{L}(e)$ is increasing in $L>0$ which completes the proof of Theorem 6.3.

Proof of Theorem 6.5. Referring to Theorem 2.4, it follows that for each $B>0$, we have:

$$
\frac{c_{\Omega, A, \sqrt{B} q, B f}^{*}(e)}{\sqrt{B}}=\min _{\lambda>0} \frac{k_{\Omega, e, A, \sqrt{B} q, B \zeta}(\lambda)}{\lambda \sqrt{B}} .
$$

Owing to the same justifications explained in the proof of Theorem 6.1, $k_{\Omega, e, A, \sqrt{B} q, B \zeta}(\lambda)$ is the first eigenvalue of the problem

$$
\left\{\begin{array}{c}
\nabla \cdot(A(y) \nabla \psi(y))+\left[\lambda^{2} e \cdot A e-\lambda \sqrt{B} q_{1}(y)+B \zeta(y)\right] \psi(y)=k_{\Omega, e, A, \sqrt{B} q, B \zeta}(\lambda) \psi \text { in } \omega, \\
\nu(x, y) \cdot A(y) \nabla \psi(y)=\left(0 ; \nu_{\omega}(y)\right) \cdot A(y) \nabla \psi(y)=0 \text { on } \mathbb{R} \times \partial \omega .
\end{array}\right.
$$

For each $\lambda>0$ and $B>0$, let $\lambda^{\prime}=\lambda / \sqrt{B}$ and $k_{\Omega, e, A, \sqrt{B} q, B \zeta}(\lambda)=\mu\left(\lambda^{\prime}, B\right)$. The first eigenvalue $\mu\left(\lambda^{\prime}, B\right)$ has the following characterization:

$$
\begin{aligned}
& \forall \lambda^{\prime}>0, \forall B>0,-\frac{\mu\left(\lambda^{\prime}, B\right)}{\lambda^{\prime} B}= \\
& \min _{\varphi \in H^{1}(C) \backslash\{0\} ;} \frac{\int_{C} \nabla \varphi \cdot A(y) \nabla \varphi d y}{\lambda^{\prime} B}+\int_{C} q_{1} \varphi^{2}-\lambda^{\prime} \int_{C} e A e \varphi^{2}-\frac{\int_{C} \zeta(y) \varphi^{2}(y) d y}{\lambda^{\prime}} \\
& \|\varphi\|_{L^{2}(C)}=1 \\
& =\min _{\varphi \in H^{1}(C) \backslash\{0\}} R\left(\lambda^{\prime}, B, \varphi\right) . \\
& \|\varphi\|_{L^{2}(C)}=1
\end{aligned}
$$

On the other hand, $B \mapsto R\left(\lambda^{\prime}, B, \varphi\right)$ is decreasing in $B>0$. Consequently, fixing $\lambda^{\prime}>0$ and taking $0<B<B^{\prime}$,

$$
\begin{aligned}
-\frac{\mu\left(\lambda^{\prime}, B\right)}{\lambda^{\prime} B}=R\left(\lambda^{\prime}, B, \psi^{\lambda^{\prime}, B}\right)>R\left(\lambda^{\prime}, B^{\prime}, \psi^{\lambda^{\prime}, B}\right) & \geq \min _{\substack{\left\|\in H^{1}(C) \backslash\{0\} ;\\
\right\| \varphi \|_{L^{2}(C)}=1}} R\left(\lambda^{\prime}, B^{\prime}, \varphi\right) \\
& =-\frac{\mu\left(\lambda^{\prime}, B^{\prime}\right)}{\lambda^{\prime} B^{\prime}} .
\end{aligned}
$$


In other words, for all $\lambda^{\prime}>0$, the function $B \mapsto \mu\left(\lambda^{\prime}, B\right) / \lambda^{\prime} B$ is increasing in $B>0$. Now, we take randomly $0<B<B^{\prime}$. Thus,

$$
\begin{aligned}
\frac{c_{\Omega, A, \sqrt{B^{\prime}} q, B^{\prime} f}^{*}(e)}{\sqrt{B^{\prime}}} & =\min _{\lambda^{\prime}>0} \frac{\mu\left(\lambda^{\prime}, B^{\prime}\right)}{\lambda^{\prime} B^{\prime}}=\frac{\mu\left(\lambda_{B^{\prime}}^{\prime}, B^{\prime}\right)}{\lambda_{B^{\prime}}^{\prime} \times B^{\prime}} \\
& >\frac{\mu\left(\lambda_{B^{\prime}}^{\prime}, B\right)}{\lambda_{B^{\prime}}^{\prime} \times B} \geq \min _{\lambda^{\prime}>0} \frac{\mu\left(\lambda^{\prime}, B\right)}{\lambda^{\prime} B}=\frac{c_{\Omega, A, \sqrt{B} q, B f}^{*}(e)}{\sqrt{B}},
\end{aligned}
$$

which means that $B \mapsto c_{\Omega, A, \sqrt{B}}^{*}, B f(e) / \sqrt{B}$ is increasing in $B>0$.

\section{Applications to homogenization problems}

The reaction-advection-diffusion problem set in a heterogenous periodic domain $\Omega$ satisfying (2.1) generates a homogenization problem:

Let $e \in \mathbb{R}^{d}$ be a vector of unit norm. Assume that $\Omega, A, q$, and $f$ are $\left(L_{1}, \ldots, L_{d}\right)$ - periodic and that they satisfy (2.1), (2.2), (2.3), (2.4) and (2.5).

For each $\varepsilon>0$, let $\Omega^{\varepsilon}=\varepsilon \Omega$ and consider the following re-scales:

$$
\forall(x, y) \in \Omega^{\varepsilon}, \quad A_{\varepsilon}(x, y)=A\left(\frac{x}{\varepsilon}, \frac{y}{\varepsilon}\right), q_{\varepsilon}(x, y)=q\left(\frac{x}{\varepsilon}, \frac{y}{\varepsilon}\right), \text { and } f_{\varepsilon}(x, y)=f\left(\frac{x}{\varepsilon}, \frac{y}{\varepsilon}\right) .
$$

The coefficients $A_{\varepsilon}, q_{\varepsilon}$, and $f_{\varepsilon}$ together with the domain $\Omega^{\varepsilon}$ are $\left(\varepsilon L_{1}, \ldots, \varepsilon L_{d}\right)$-periodic, and they satisfy similar properties to those of $A, q, f$ and $\Omega$.

Consider the parametric reaction-advection-diffusion problem

$$
\left(P_{\varepsilon}\right)\left\{\begin{array}{l}
u_{t}^{\varepsilon}(t, x, y)=\nabla \cdot\left(A_{\varepsilon} \nabla u^{\varepsilon}\right)(t, x, y)+q_{\varepsilon} \cdot \nabla u^{\varepsilon}+f_{\varepsilon}\left(x, y, u^{\varepsilon}\right), t \in \mathbb{R},(x, y) \in \Omega^{\varepsilon}, \\
\nu^{\varepsilon} \cdot A_{\varepsilon} \nabla u^{\varepsilon}(t, x, y)=0, \quad t \in \mathbb{R},(x, y) \in \partial \Omega^{\varepsilon},
\end{array}\right.
$$

where $\nu^{\varepsilon}(x, y)$ denotes the outward unit normal on $\partial \Omega^{\varepsilon}$ at the point $(x, y)$.

Owing to the results found by Berestycki and Hamel in section 6 of [2], and since the coefficients $A_{\varepsilon}, f_{\varepsilon}$ and $q_{\varepsilon}$ together with the domain $\Omega^{\varepsilon}$ satisfy all the necessary assumptions, it follows that the problem $\left(P_{\varepsilon}\right)$ admits a minimal speed of propagation $c_{\Omega^{\varepsilon}}^{*}, A_{\varepsilon}, q_{\varepsilon}, f_{\varepsilon}(e)>0$ such that $\left(P_{\varepsilon}\right)$ has a solution $u^{\varepsilon}$ in the form of a pulsating front within a speed $c$ if and only if $c \geq c_{\Omega^{\varepsilon}}^{*}, A_{\varepsilon}, q_{\varepsilon}, f_{\varepsilon}(e)>0$.

In this section, we investigate the limit of the parametric minimal speeds $c_{\Omega^{\varepsilon}}^{*}, A_{\varepsilon}, q_{\varepsilon}, f_{\varepsilon}(e)$ (whose parameter is $\varepsilon$ ) of the problems $\left(P_{\varepsilon}\right)_{\varepsilon>0}$ as $\varepsilon \rightarrow 0^{+}$. In other words, we search the limit of these minimal speeds as the periodicity cell $C^{\varepsilon}=\varepsilon C$ becomes a very small size. On the other hand, we study although not the most general setting, the variation of the map $\varepsilon \mapsto c_{\Omega^{\varepsilon}, A_{\varepsilon}, q_{\varepsilon}, f_{\varepsilon}}(e)$ in $\varepsilon>0$.

Theorem 8.1 Let $e \in \mathbb{R}^{d}$ be a unit vector, and let $\Omega \subseteq \mathbb{R}^{N}$ be a domain which is $L$-periodic and satisfying (2.1). Assume that $A=A(x, y), q=q(x, y)$, and $f=f(x, y, u)$ are $L$-periodic and that they satisfy (2.2), (2.3), (2.4) and (2.5) together with the assumptions $\nabla . A \tilde{e} \equiv 0$ on $\bar{\Omega}$ and $\nu . A \tilde{e}=0$ on $\partial \Omega$. For each $\varepsilon>0$, consider the problem

$$
\left\{\begin{array}{l}
u_{t}^{\varepsilon}(t, x, y)=\nabla \cdot\left(A_{\varepsilon} \nabla u^{\varepsilon}\right)(t, x, y)+q_{\varepsilon} \cdot \nabla u^{\varepsilon}+f_{\varepsilon}\left(x, y, u^{\varepsilon}\right), t \in \mathbb{R},(x, y) \in \Omega^{\varepsilon}, \\
\nu^{\varepsilon} \cdot A_{\varepsilon} \nabla u^{\varepsilon}(t, x, y)=0, \quad t \in \mathbb{R},(x, y) \in \partial \Omega^{\varepsilon},
\end{array}\right.
$$


where $A_{\varepsilon}, f_{\varepsilon}$ and $q_{\varepsilon}$ are the coefficients defined in the beginning of this section. Then, the minimal speed $c_{\Omega^{\varepsilon}}^{*}, A_{\varepsilon}, q_{\varepsilon}, f_{\varepsilon}(e)$ of pulsating travelling fronts propagating in the direction of $e$ and solving 8.1) satisfies

$$
\lim _{\varepsilon \rightarrow 0^{+}} c_{\Omega^{\varepsilon}}^{*}, A_{\varepsilon}, q_{\varepsilon}, f_{\varepsilon}(e)=2 \sqrt{f_{C} \tilde{e} A \tilde{e}(x, y) d x d y} \sqrt{f_{C} \zeta(x, y) d x d y}
$$

where $C$ is the periodicity cell of $\Omega$ and $\tilde{e}=(e, 0, \cdots, 0) \in \mathbb{R}^{N}$.

Proof. As a first notice, we mention that the domain $\Omega^{\varepsilon}$ is the image of $\Omega$ by the a dilation whose center is the origin $O(0, \ldots, 0)$ and whose scale factor is equal to $\varepsilon$. Consequently,

$$
\begin{gathered}
\text { for each } \varepsilon>0,(\varepsilon x, \varepsilon y) \in \Omega^{\varepsilon} \quad \text { if and only if } \quad(x, y) \in \Omega \text {, and } \\
(\varepsilon x, \varepsilon y) \in \partial \Omega^{\varepsilon} \quad \text { if and only if } \quad(x, y) \in \partial \Omega .
\end{gathered}
$$

Moreover,

$$
\forall \varepsilon>0, \forall(x, y) \in \partial \Omega, \nu^{\varepsilon}(\varepsilon x, \varepsilon y)=\nu(x, y) .
$$

Consider now, for each $\varepsilon>0$, the following change of variables

$$
v^{\varepsilon}(t, x, y)=u^{\varepsilon}(t, \varepsilon x, \varepsilon y) ; \quad(t, x, y) \in \mathbb{R} \times \Omega .
$$

One gets

$$
\begin{gathered}
\forall(t, x, y) \in \mathbb{R} \times \Omega, v_{t}^{\varepsilon}(t, x, y)=u_{t}^{\varepsilon}(t, \varepsilon x, \varepsilon y), \\
\nabla_{x, y} \cdot\left(A(x, y) \nabla v^{\varepsilon}\right)(t, x, y)=\nabla_{x, y} \cdot\left(A_{\varepsilon} \nabla u^{\varepsilon}\right)(t, \varepsilon x, \varepsilon y)=\varepsilon^{2} \nabla \cdot\left(A_{\varepsilon} \nabla u^{\varepsilon}\right)(t, \varepsilon x, \varepsilon y),
\end{gathered}
$$

and

$$
\begin{aligned}
\nu_{\varepsilon}(\varepsilon x, \varepsilon y) \cdot\left[A_{\varepsilon} \nabla u^{\varepsilon}\right](t, \varepsilon x, \varepsilon y) & =\nu(x, y) \cdot A\left(\frac{\varepsilon x}{\varepsilon}, \frac{\varepsilon y}{\varepsilon}\right) \nabla u^{\varepsilon}(t, \varepsilon x, \varepsilon y) \\
& =\frac{1}{\varepsilon} \nu(x, y) \cdot A(x, y) \nabla v^{\varepsilon}(t, x, y) \text { on } \mathbb{R} \times \partial \Omega
\end{aligned}
$$

The boundary condition in (8.1) yields that $\nu_{\varepsilon}(\varepsilon x, \varepsilon y) \cdot\left[A_{\varepsilon} \nabla u^{\varepsilon}\right](t, \varepsilon x, \varepsilon y)=0$, for all $(t, x, y) \in$ $\mathbb{R} \times \partial \Omega$ (which is equivalent to say: for all $\left.(t, \varepsilon x, \varepsilon y) \in \mathbb{R} \times \partial \Omega^{\varepsilon}\right)$. It follows from (8.3) that

$$
\forall(t, x, y) \in \mathbb{R} \times \partial \Omega, \quad \nu \cdot A \nabla v^{\varepsilon}(t, x, y)=0 .
$$

One can now conclude that: for each $\varepsilon>0, u^{\varepsilon}$ satisfies (3.1) if and only if $v^{\varepsilon}$ satisfies

$$
\left\{\begin{array}{l}
v_{t}^{\varepsilon}(t, x, y)=\frac{1}{\varepsilon^{2}} \nabla \cdot\left(A \nabla v^{\varepsilon}\right)(t, x, y)+\frac{1}{\varepsilon} q \cdot \nabla v^{\varepsilon}+f\left(x, y, v^{\varepsilon}\right), t \in \mathbb{R},(x, y) \in \Omega, \\
\nu \cdot A \nabla v^{\varepsilon}(t, x, y)=0, t \in \mathbb{R},(x, y) \in \partial \Omega .
\end{array}\right.
$$

Having the assumptions (2.1), 2.2), (2.3), (2.4), and (2.5) on $\Omega, A, q$, and $f$, one gets that problem (8.4) admits, for each $\varepsilon>0$, a minimal speed of propagation denoted by $c_{\Omega,\left(\frac{1}{\varepsilon}\right)^{2} A, \frac{1}{\varepsilon} q, f}(e)$.

Moreover, due to the change of variables between $u^{\varepsilon}$ and $v^{\varepsilon}$, it follows that for each $\varepsilon>0, u^{\varepsilon}$ is a pulsating travelling front propagating in the direction of $e$ within a speed $c$ and solving (8.1) 
if and only if $v^{\varepsilon}$ is a pulsating travelling front propagating in the direction of $e$ within a speed $\frac{c}{\varepsilon}$ and solving (8.4). This yields that

$$
\forall \varepsilon>0, \quad c_{\Omega^{\varepsilon}}^{*}, A_{\varepsilon}, q_{\varepsilon}, f_{\varepsilon}(e)=\varepsilon c_{\Omega,\left(\frac{1}{\varepsilon}\right)^{2} A, \frac{1}{\varepsilon} q, f}(e)=c_{\Omega, M A, \sqrt{M} q, f}^{*}(e) / \sqrt{M},
$$

where $M=(1 / \varepsilon)^{2}$.

As $\varepsilon \rightarrow 0^{+}$, the variable $M \rightarrow+\infty$. Applying Theorem 4.1, with $\gamma=\frac{1}{2}$, one gets that

$$
\lim _{M \rightarrow+\infty} \frac{c_{\Omega, M A, \sqrt{M} q, f}^{*}(e)}{\sqrt{M}}=2 \sqrt{f_{C} \tilde{e} A \tilde{e}(x, y) d x d y} \sqrt{f_{C} \zeta(x, y) d x d y}
$$

Therefore, $\lim _{\varepsilon \rightarrow 0^{+}} c_{\Omega^{\varepsilon}}^{*}, A_{\varepsilon}, q_{\varepsilon}, f_{\varepsilon}(e)=2 \sqrt{f_{C} \tilde{e} A \tilde{e}(x, y) d x d y} \sqrt{f_{C} \zeta(x, y) d x d y}$, and the proof of Theorem 8.1 is complete.

Remark 8.2 It is worth noticing that, in formula 8.9, the homogenized speed depends on the averages of the diffusion and reaction coefficients, but it does not depend on the advection.

We move now to study the variation of the map $\varepsilon \mapsto c_{\Omega^{\varepsilon}}^{*}, A_{\varepsilon}, q_{\varepsilon}, f_{\varepsilon}(e)$ with respect to $\varepsilon>0$. In other words, we want to check the monotonicity behavior of the parametric minimal speed of propagation, whose parameter $\varepsilon>0$, as the periodicity cell of the domain of propagation shrinks or enlarges within a ratio $\varepsilon$. In this study, we will consider the same situation of Theorem 6.1 and also the same notations introduced in the beginning of section 8:

Theorem 8.3 Let $e=(1,0 \ldots, 0)$. Assume that $\Omega$ has the form $\mathbb{R} \times \omega$ where $\omega$ may or may not

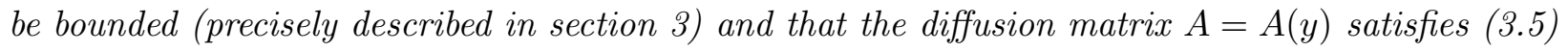
together with the assumption that $e$ is an eigenvector of $A(y)$ for all $y \in \bar{\omega}$, that is

$$
A(x, y) e=A(y) e=\alpha(y) e, \text { for all }(x, y) \in \mathbb{R} \times \bar{\omega}
$$

where $y \mapsto \alpha(y)$ is a positive $\left(L_{1}, \ldots, L_{d}\right)$ - periodic function defined over $\bar{\omega}$. The nonlinearity $f$ is assumed to satisfy (3.5) and (3.4). Assume further more that the advection field $q$ (when it exists) is in the form $q(x, y)=\left(q_{1}(y), 0, \ldots, 0\right)$ where $q_{1}$ has a zero average over $C$, the periodicity cell of $\omega$. For $\varepsilon>0$ consider the reaction-advection-diffusion problem

$$
\left\{\begin{array}{l}
\forall t \in \mathbb{R}, \forall(x, y) \in \Omega^{\varepsilon}=\mathbb{R} \times \varepsilon \omega \\
u_{t}^{\varepsilon}(t, x, y)=\nabla \cdot\left(A_{\varepsilon} \nabla u^{\varepsilon}\right)(t, x, y)+q_{\varepsilon} \cdot \nabla u^{\varepsilon}+f_{\varepsilon}\left(x, y, u^{\varepsilon}\right) \\
\nu^{\varepsilon} \cdot A_{\varepsilon} \nabla u^{\varepsilon}(t, x, y)=0, t \in \mathbb{R},(x, y) \in \partial \Omega^{\varepsilon}
\end{array}\right.
$$

Then, the map $\varepsilon \mapsto c_{\Omega^{\varepsilon}}^{*}, A_{\varepsilon}, q_{\varepsilon}, f_{\varepsilon}(e)$ is increasing in $\varepsilon>0$.

Proof of Theorem 8.3. For each $\varepsilon>0$, we consider the change of variables

$$
v^{\varepsilon}(t, x, y)=u^{\varepsilon}(t, \varepsilon x, \varepsilon y) ; \quad(t, x, y) \in \mathbb{R} \times \Omega .
$$


Owing to the justifications shown in the proof of Theorem 8.1, one consequently obtains

$$
\forall \varepsilon>0, \quad c_{\Omega^{\varepsilon}}^{*}, A_{\varepsilon}, q_{\varepsilon}, f_{\varepsilon}(e)=\varepsilon c_{\Omega,\left(\frac{1}{\varepsilon}\right)^{2} A, \frac{1}{\varepsilon} q, f}^{*}(e)=c_{\Omega, \beta A, \sqrt{\beta} q, f}^{*}(e) / \sqrt{\beta},
$$

where $\beta(\varepsilon)=(1 / \varepsilon)^{2}$.

Applying Theorem 6.1, it follows that the map $\eta_{1}: \beta \mapsto c_{\Omega, \beta A, \sqrt{\beta} q, f}^{*}(e) / \sqrt{\beta}$ is decreasing in $\beta>0$. On the other hand, the map $\eta_{2}: \varepsilon \mapsto \beta(\varepsilon)$ is also decreasing in $\varepsilon>0$. Therefore, $\varepsilon \mapsto c_{\Omega^{\varepsilon}}^{*}, A_{\varepsilon}, q_{\varepsilon}, f_{\varepsilon}(e)$, which is the composition $\eta_{1} \circ \eta_{2}$, is increasing in $\varepsilon>0$ and this completes our proof.

Other homogenization results, concerning reaction-advection-diffusion problems, were given in the case of a combustion-type nonlinearity $f=f(u)$ satisfying

$$
\left\{\begin{array}{l}
\exists \theta \in(0,1), f(s)=0 \text { for all } s \in[0, \theta], f(s)>0 \text { for all } s \in(\theta, 1), f(1)=0, \\
\exists \rho \in(0,1-\theta), \quad f \text { is non-increasing on }[1-\rho, 1]
\end{array}\right.
$$

Consider the equation

$$
u_{t}^{\varepsilon}(t, x)=\nabla \cdot\left(A\left(\varepsilon^{-1} x\right) \nabla u^{\varepsilon}\right)+\varepsilon^{-1} q\left(\varepsilon^{-1} x\right) \cdot \nabla u^{\varepsilon}+f\left(u^{\varepsilon}\right) \quad \text { in } \mathbb{R}^{N},
$$

where the nonlinearity $f$ satisfies (8.9), and the drift and diffusion coefficients $q$ and $A$ satisfy the general assumptions (2.2) and (2.3), with periodicity 1 in all variables $x_{1}, \ldots, x_{N}$. Fix a unit vector $e$ of $\mathbb{R}^{N}$. From Berestycki and Hamel [2], it follows that for each $\varepsilon>0$, problem (8.10) admits a unique pulsating front $\left(c_{\varepsilon}, u^{\varepsilon}\right)$ such that

$$
u^{\varepsilon}(t, x)=\phi^{\varepsilon}\left(x \cdot e+c_{\varepsilon} t, x\right)
$$

where $\phi^{\varepsilon}(s, x)$ is $(\varepsilon, \ldots, \varepsilon)$-periodic in $x$ that satisfies $\phi^{\varepsilon}(-\infty,)=$.0 and $\phi^{\varepsilon}(+\infty,)=$.1 . The functions $u^{\varepsilon}$ are actually unique up to shifts in time, and one can assume that $\max _{\mathbb{R}^{N}} \phi^{\varepsilon}(0,)=.\theta$.

Concerning problem (8.10), Heinze [15] proved that

$$
\text { as } \varepsilon \rightarrow 0^{+}, c_{\varepsilon} \rightarrow c_{0}>0 \text {, and } u^{\varepsilon}(t, x) \rightarrow u_{0}\left(x \cdot e+c_{0} t\right) \text { weakly in } H_{l o c}^{1},
$$

where $\left(c_{0}, u_{0}\right)$ is the unique solution of the one-dimensional homogenized equation

$$
\left\{\begin{array}{l}
a^{*} u_{0}^{\prime \prime}-c_{0} u_{0}^{\prime}+f\left(u_{0}\right)=0 \text { in } \mathbb{R} \\
u_{0}(-\infty)=0<u_{0}<u_{0}(+\infty)=1 \text { in } \mathbb{R}, u_{0}(0)=\theta
\end{array}\right.
$$

and $a^{*}$ is a positive constant determined in 15.

In Theorem 1 of Caffarelli, Lee, Mellet [10], the homogenization limit was combined with the singular high activation limit for the reaction (one can also see [11] in this context) while the diffusion matrix was taken $A=I d_{\mathbb{R}^{N}}$. More precisely, the nonlinearity had the form $f_{\varepsilon}(u)=\frac{1}{\varepsilon} \beta\left(\frac{u}{\varepsilon}\right)$ with $\beta(s)$ a Lipschitz fucntion satisfying

$$
\beta(s)>0 \text { in }(0,1) \text { and } \beta(s)=0 \text { otherwise. }
$$

These nonlinearities approach a Dirac mass at $u=1$. 


\section{Open problems}

In all the results of this paper, we deal with nonlinearities of the "KPP" type. In the periodic framework of this paper, pulsating travelling fronts exist also with other types of nonlinearities (see Theorems 1.13 and 1.14 in [2]). Namely, they exist when $f=f(x, y, u)$ is of the "combustion" type satisfying:

$$
\left\{\begin{array}{l}
f \text { is globally Lipschitz-continuous in } \bar{\Omega} \times \mathbb{R}, \\
\forall(x, y) \in \bar{\Omega}, \forall s \in(-\infty, 0] \cup[1,+\infty), f(s, x, y)=0, \\
\exists \rho \in(0,1), \forall(x, y) \in \bar{\Omega}, \forall 1-\rho \leq s \leq s^{\prime} \leq 1, f(x, y, s) \geq f\left(x, y, s^{\prime}\right),
\end{array}\right.
$$

and

$$
\left\{\begin{array}{l}
f \text { is } L \text {-periodic with respect to } x, \\
\exists \theta \in(0,1), \forall(x, y) \in \bar{\Omega}, \forall s \in[0, \theta], f(x, y, s)=0, \\
\forall s \in(\theta, 1), \exists(x, y) \in \bar{\Omega} \text { such that } f(x, y, s)>0,
\end{array}\right.
$$

or when $f=f(x, y, u)$ is of the "ZFK" (for Zeldovich-Frank- Kamenetskii) type satisfying (9.1) and

$$
\left\{\begin{array}{l}
f \text { is } L \text {-periodic with respect to } x, \\
\exists \delta>0, \text { the restriction of } f \text { to } \bar{\Omega} \times[0,1] \text { is of class } C^{1, \delta}, \\
\forall s \in(0,1), \exists(x, y) \in \bar{\Omega} \text { such that } f(x, y, s)>0 .
\end{array}\right.
$$

In particular, the "KPP" nonlinearities are of the "ZFK" type.

Recently, El Smaily [12] gave min - max and max - min formulæ for the speeds of propagation of problem (2.6) taken with a "ZFK" or a "combustion" nonlinearity. These formulæ, together with the results of this paper, can give important estimates for the parametric minimal speeds of the problem (2.6) when $f$ is a "ZFK" nonlinearity which is not of the "KPP" type. Indeed, if $f$ is a "ZFK" nonlinearity, one can find a "KPP" function $h=h(x, y, u)$ such that

$$
\forall(x, y, u) \in \bar{\Omega} \times \mathbb{R}, f(x, y, u) \leq h(x, y, u) .
$$

Referring to formula (1.17) in El Smaily [12], one can conclude that

$$
\forall M>0, \forall B>0, \forall \gamma \in \mathbb{R}, \quad c_{\Omega, M A, M^{\gamma}}^{*}, B f^{(e)} \leq c_{\Omega, M A}^{*} M^{\gamma} q, B h^{(e)} .
$$

Moreover, if $f$ is a "ZFK" nonlinearity satisfying the additional assumption

$$
\forall(x, y) \in \bar{\Omega}, f_{u}^{\prime}(x, y, 0)>0,
$$

then one can find a "KPP" function $g=g(x, y, u)$ such that $g \leq f$ in $\bar{\Omega} \times \mathbb{R}$, and thus

$$
\begin{gathered}
\forall M>0, \forall B>0, \forall \gamma \in \mathbb{R}, \\
c_{\Omega, M A, M^{\gamma} q, B g}^{(e)} \leq c_{\Omega, M A, M^{\gamma} q, B f^{*}}^{(e)} \leq c_{\Omega, M A, M^{\gamma} q, B h^{*}}^{(e) .}
\end{gathered}
$$


As a consequence, under the assumptions that $0 \leq \gamma \leq 1 / 2, \nu \cdot A \tilde{e}=0$ on $\partial \Omega$, and $\nabla \cdot A \tilde{e} \equiv 0$ in $\Omega$, Theorem 4.1 implies that

$$
\limsup _{M \rightarrow+\infty} \frac{c_{\Omega, M A, M^{\gamma} q, f}^{*}(e)}{\sqrt{M}} \leq 2 \sqrt{f_{C} \tilde{e} A \tilde{e}(x, y) d x d y} \sqrt{f_{C} g_{u}^{\prime}(x, y, 0) d x d y}
$$

and

$$
\liminf _{M \rightarrow+\infty} \frac{c_{\Omega, M A, M^{\gamma} q, f}^{*}(e)}{\sqrt{M}}=2 \sqrt{f_{C} \tilde{e} A \tilde{e}(x, y) d x d y} \sqrt{f_{C} h_{u}^{\prime}(x, y, 0) d x d y}>0 .
$$

If $f$ is a "combustion" nonlinearity, then problem (2.6) admits a solution $(c, u)$ where $c=$ $c_{\Omega, A, q, f}(e)>0$ is unique and $u=u(t, x, y)$ is increasing in $t$ and it is unique up to a translation in $t$. Taking $g$ as a "KPP" nonlinearity such that $g \geq f$ in $\bar{\Omega} \times \mathbb{R}$ and using Theorem 4.1, it follows that

$$
\begin{aligned}
& \limsup _{M \rightarrow+\infty} \frac{c_{\Omega, M A, M \gamma_{q, f}}(e)}{\sqrt{M}} \leq 2 \sqrt{f_{C} \tilde{e} A \tilde{e}(x, y) d x d y} \sqrt{f_{C} g_{u}^{\prime}(x, y, 0) d x d y} \\
& \text { together with } \liminf _{M \rightarrow+\infty} \frac{c_{\Omega, M A, M \gamma}(e)}{\sqrt{M}} \geq 0 .
\end{aligned}
$$

Similarly, one can get several estimates concerning the case of a small diffusion factors, small (resp. large) reaction factors, or small (resp. large) periodicity parameters.

The above motivation gives several upper and lower estimates for the parametric speeds of propagation. However, the exact limits are not known. This leads us to ask about the asymptotics of the minimal speeds of propagation with respect to diffusion, reaction and periodicity factors in the "ZFK" case and about the asymptotics of the unique parametric speed of propagation in the "combustion" case. These studies should help, as it was done in section 8 , in solving some homogenization problems in the "ZFK" case.

Besides, Theorem 8.1 gives the limit of $c_{\Omega^{\varepsilon}}^{*}, A_{\varepsilon}, q_{\varepsilon}, f_{\varepsilon}(e)$ as $\varepsilon \rightarrow 0^{+}$. However, finding the homogenized equation of (8.1) in the "KPP" remains an open problem.

\section{Conclusions}

As we mentioned in the beginning of this paper, our first aim was to give a complete and rigorous analysis of the minimal speed of propagation of pulsating travelling fronts solving parametric heterogeneous reaction-advection-diffusion equations in a periodic framework. In the paper of Berestycki, Hamel and Nadirashvili [3], several upper and lower estimates for the parametric minimal speed of propagation were given (see Theorems 1.6 and 1.10 in [3]). However, the exact asymptotic behaviors of the minimal speed with respect to diffusion and reaction factors and with respect to the periodicity parameter $L$ were not given there. In this paper, we determined the exact asymptotes of the minimal speed in the "KPP" periodic framework. In sections 3 , 4 and 5 , we proved that (under some assumptions on $A, q, f$ and $\Omega$ ) the asymptotes of the parametric minimal speed are either

$$
2 \sqrt{\max _{\bar{\omega}} \zeta} \sqrt{\max _{\bar{\omega}} e A e} \quad \text { or } \quad 2 \sqrt{f_{C} \tilde{e} A \tilde{e}(x, y) d x d y} \sqrt{f_{C} \zeta(x, y) d x d y} .
$$

(see Theorems 3.1, 3.4, 4.1, 4.3, 5.1 and 5.2 above). Moreover, we found in section 3 that the presence of an advection field, in the general form or in the form of shear flows, changes the 
asymptotic behavior of the minimal speed within a small diffusion (see Theorem 3.3 and Remark 3.6). Conversely, we proved in Section 4 that the presence of a general advection field $M^{\gamma} q$ (where $q$ satisfies (2.3)) has no effect on $\lim _{M \rightarrow+\infty} \frac{c_{\Omega, M A}^{*} M^{\gamma} q, f^{(e)}}{\sqrt{M}}$ whenever $0 \leq \gamma \leq 1 / 2$ (see Theorem 4.1). Furthermore, we studied, in a particular periodic framework, the variations of the maps $\beta \mapsto \frac{c_{\Omega, \beta A, \sqrt{\beta} q, f}^{*}(e)}{\sqrt{\beta}}$ and $L \mapsto c_{\mathbb{R}^{N}, A_{L}, q_{L}, f_{L}}^{*}(e)$ and $B \mapsto \frac{c_{\Omega, A, \sqrt{B} q, B f}^{*}(e)}{\sqrt{B}}$ with respect to the positive variables $\beta, L$ and $B$ respectively. Roughly speaking, we found that the first and the third maps have opposite senses of variations (see Theorems 6.1 and 6.5). On the other hand, Theorem 6.3 and Theorem 8.3 yield that the minimal speed increases when the medium undergoes a dilation whose scale factor is greater than 1.

The second aim was to find the homogenized "KPP" minimal speed. We achieved this goal in section 8 (Theorem 8.1) under the assumptions of free divergence on $A(x, y) \tilde{e}$ and invariance of the domain in the direction $A(x, y) \tilde{e}$. This was an application to the results obtained in section 1 . The found homogenized speed should play an important role in finding the homogenized reactionadvection-diffusion equation in the "KPP" case. In a forthcoming paper [13], we find also the homogenized speed in the one dimensional case but in a more general setting (in fact, the assumption of divergence free is equivalent to the assumption that the diffusion term $x \mapsto a(x)$ is constant over $\mathbb{R}$ in the case $N=1$ ).

All the mathematical results obtained in this paper can be applied to study some spreading phenomena. Referring to the results of Weinberger [30], one can conclude that the spreading speed is equal to the "KPP" minimal speed of propagation in the periodic framework under some assumptions on the initial data $u_{0}:=u_{0}(x, y)=u(0, x, y)$ which is defined on a periodic domain $\Omega$ of $\mathbb{R}^{N}$. In such a setting, all our results can be applied to give rigorous answers on the asymptotic behavior of the parametric spreading speed with respect to diffusion and reaction factors and with respect to the periodicity parameter.

\section{Acknowledgments}

I am very grateful to Professor François Hamel for his valuable comments, directions and advices. I would like also to thank Professor Mustapha Jazar for his support and his constant encouragement during the preparation of this work.

\section{References}

[1] H. Berestycki, The influence of advection on the propagation of fronts in reaction-diffusion equations, In: Nonlinear PDEs in Condensed Matter and Reactive Flows, H. Berestycki and Y. Pomeau eds., Kluwer Academic Publ., 2002, pp 1-45.

[2] H. Berestycki, F. Hamel, Front propagation in periodic excitable media, Comm. Pure Appl. Math.55 (2002), pp 949-1032.

[3] H. Berestycki, F. Hamel, N.Nadirashvili, The Speed of Propagation for KPP Type Problems (Periodic Framework), J. Eur. Math. Soc. 7 (2005), pp 173-213. 
[4] H. Berestycki, F. Hamel, N.Nadirashvili, Elliptic Eigenvalue Problems with Large Drift and Applications to Nonlinear Propagation Phenomena, Comm. Math. Phys. 253 (2005), 2, 451-480.

[5] H. Berestycki, F. Hamel, L. Roques, Analysis of the periodically fragmented environment model : I - The effect of heterogeneous environnement on species conservations, J. Math. Biol. 51 (2005), no. 1 , pp 75-113.

[6] H. Berestycki, F. Hamel, L. Roques, Analysis of the periodically fragmented environment model : II - Biological invasions and pulsating travelling fronts, J. Math. Pures Appl. (9) 84 (2005), no. 8, pp 1101-1146.

[7] H. Berestycki, L. Nirenberg, On the method of moving planes and the sliding method, Bol. Soc. Bras. Mat, 22(1991), pp 1-37.

[8] H. Berestycki, L. Nirenberg, Travelling fronts in cylinders, Ann. Inst. H. Poincaré, Anal. Non Lin. 9 (1992), pp 1-37.

[9] H. Berestycki, L. Nirenberg and S.R.S. Varadhan, The principal eigenvalue and maximum principle for second order elliptic operators in general domains, Comm. Pure Appl. Math. 47 (1994), pp 47-92.

[10] L. A. Caffarelli, K.-A. Lee, A. Mellet, Singular limit and homogenization for flame propagation in periodic excitable media, Arch. Ration. Mech. Anal. 172 (2004), no. 2, 153-190.

[11] L. A. Caffarelli, K.-A. Lee, A. Mellet, Homogenization and flame propagation in periodic excitable media: the asymptotic speed of propagation, Comm. Pure Appl. Math. 59 (2006), no. 4, 501-525.

[12] M. El Smaily, Min-Max formulae for the speeds of pulsating travelling fronts in periodic excitable media, submitted.

[13] M. El Smaily, F. Hamel, L. Roques, Homogenization and influence of fragmentation in a biological invasion model, (2007), to appear in Discrete Contin. Dyn. Syst.

[14] P.C. Fife, Mathematical aspects of reacting and diffusing systems, Lecture Notes in Biomathematics 28, Springer Verlag, Berlin-New York, 1979.

[15] S. Heinze, Homogenization of flame fronts, Preprint IWR, Heidelberg, 1993.

[16] S. Heinze, Large convection limits for KPP fronts, Preprint 21/(2005), Max-Planck Institut für Mathimatik in den Naturwissenschaften, Leipzig.

[17] N. Kinezaki, K. Kawasaki, N. Shigesada, Spatial dynamics of invasion in sinusoidally varying enviroments, Popul Ecol 48 (2006), pp 263-270.

[18] N. Kinezaki, K. Kawasaki, F. Takasu, N. Shigesada, Modeling biological invasions into periodically fragmented environments, Theor. Population Biology 64, (2003), pp 291-302.

[19] A.N Kolmogorov, I.G Petrovsky, N.S Piskunov, Étude de l'équation de la diffusion avec croissance de la quantité de matiére et son application a un probléme biologique, Bulletin Université d'Etat à Moscou (Bjul. Moskowskogo Gos. Univ.), Série internationale A1 (1937), pp 1-26. 
[20] J.D. Murray Mathematical biology. I. An introduction. Third edition. Interdisciplinary Applied Mathematics, 17. Springer-Verlag, New York, (2002).

[21] G. Nadin, Existance and uniqueness of the solution of a space-time periodic reaction-diffusion equation, (2007), preprint.

[22] G. Nadin, Travelling fronts in space-time periodic media, (2007), preprint.

[23] J. Nolen, J. Xin, Reaction-diffusion front speeds in spatially-temporally periodic shear flows, SIAM J. Multiscale Modeling and Simulation, Vol. 1, No. 4, pp. 554-570, 2003.

[24] J. Nolen, J. Xin, Existence of KPP Type Fronts in Space-Time Periodic Shear Flows and a Study of Minimal Speeds Based on Variational Principle, Discrete and Continuous Dynamical Systems, 2005, Vol. 13, No. 5, pp 1217-1234.

[25] J. Nolen, M. Rudd, J. Xin, Existence of KPP fronts in spatially-temporally periodic advection and variational principle for propagation speeds, Dynamics of PDE, Vol. 2, No. 1, pp 1-24, 2005.

[26] G. Papanicolaou, X. Xin, Reaction-diffusion fronts in periodically layered media, J. Stat. Phys. 63 (1991), pp 915-931.

[27] P. Ronney, Some open issues in premixed turbulent combustion, In: Mathematical modelling in combustion science, J. Buckmaster and T. Takeno Eds, Lect. Notes Phys., Springer-Verlag, Berlin, 1995, pp 3-22.

[28] N. Shigesada, K. Kawasaki, Biological invasions: Theory and practice, Oxford Series in Ecology and Evolution, Oxford : Oxford UP, 1997.

[29] N. Shigesada, K. Kawasaki, E. Teramoto, Spatial segregation of interacting species, J. Theoret. Biol. 79 (1979), pp 83-99.

[30] H.F Weinberger, On spreading speeds and traveling waves for growth and migration models in a periodic habitat, J. Math. Biol. 45 (2002), no. 6, pp 511-548.

[31] F. Williams, Combustion Theory, Addison-Wesley, Reading MA, 1983.

[32] X. Xin, Existence and uniqueness of travelling waves in a reaction-diffusion equation with combustion nonlinearity, Indiana Univ. Math. J. 40 (1991), pp 985-1008.

[33] X. Xin, Existence and stability of travelling waves in periodic media governed by a bistable nonlinearity, J. Dyn. Diff. Eq. 3 (1991), pp 541-573.

[34] X. Xin, Existence of planar flame fronts in convective-diffusive periodic media, Arch. Ration. Mech. Anal. 121 (1992), pp 205-233.

[35] J.X. Xin, Analysis and modeling of front propagation in heterogeneous media, SIAM Review 42 (2000), pp 161-230.

[36] J.X. Xin, Front propagation in heterogeneous media, SIAM Review 42 (2000), pp 161-230.

[37] Y.B. Zeldovich, G.I. Barenblatt, V.B. Libovich, G.M. Mackviladze, The mathematical theory of combustion and explosions, Cons. Bureau, New York, 1985. 